\title{
Inviscid limit for the axisymmetric stratified Navier-Stokes system
}

\author{
Samira Sulaiman
}

\begin{abstract}
This paper is devoted to the study of the Cauchy problem for the stratified Navier-Stokes system in three-dimensional space. In the first part of the paper, we prove the existence of a unique global solution $\left(v_{\nu}, \rho_{\nu}\right)$ for this system with axisymmetric initial data belonging to the Sobolev space $H^{s} \times H^{s-2}$ with $s>5 / 2$. The bounds on the solution are uniform with respect to the viscosity. In the second part, we analyse the inviscid limit problem. We prove that the viscous solutions $\left(v_{\nu}, \rho_{\nu}\right)_{\nu>0}$ converge strongly in the space $L_{\mathrm{loc}}^{\infty}\left(\mathbb{R}_{+} ; H^{s} \times H^{s-2}\right)$ to the solution $(v, \rho)$ of the stratified Euler system.
\end{abstract}

\section{Introduction and main results}

In this paper, we consider the incompressible stratified Navier-Stokes system in three-dimensional space

$$
\left\{\begin{array}{l}
\partial_{t} v_{\nu}+v_{\nu} \cdot \nabla v_{\nu}-\nu \Delta v_{\nu}+\nabla p_{\nu}=\rho_{\nu} e_{z}, \quad(t, x) \in \mathbb{R}_{+} \times \mathbb{R}^{3} \\
\partial_{t} \rho_{\nu}+v_{\nu} \cdot \nabla \rho_{\nu}-\Delta \rho_{\nu}=0 \\
\operatorname{div} v_{\nu}=0 \\
\left(v_{\nu}, \rho_{\nu}\right)_{\mid t=0}=\left(v^{0}, \rho^{0}\right) .
\end{array}\right.
$$

Here, the vector field $v_{\nu}=\left(v_{\nu}^{1}, v_{\nu}^{2}, v_{\nu}^{3}\right)$ stands for the velocity of the fluid and it is assumed to be divergence-free, while the scalar function $\rho_{\nu}$ denotes the density or the temperature. The pressure $p_{\nu}$ is a scalar function given by the equation

$$
\Delta p_{\nu}=-\operatorname{div}\left(v_{\nu} \cdot \nabla v_{\nu}\right)+\partial_{z} \rho_{\nu}
$$

The parameter $\nu>0$ is the kinematic viscosity, and the vector $e_{z}$ denotes the vector $(0,0,1)$. Note that the usual incompressible Navier-Stokes system arises as

Mathematics Subject Classification (2010): 76D05, 35Q30, 35S50, 76Dxx, 76D50.

Keywords: Navier-Stokes equations, paradifferential operators, incompressible viscous fluids, stratification effects in viscous fluids. 
a particular case of (1.1): it suffices to take $\rho_{\nu}=$ constant. It reads as follows:

$$
\left\{\begin{array}{l}
\partial_{t} v_{\nu}+v_{\nu} \cdot \nabla v_{\nu}-\nu \Delta v_{\nu}+\nabla p_{\nu}=0 \\
\operatorname{div} v_{\nu}=0 \\
v_{\nu \mid t=0}=v^{0}
\end{array}\right.
$$

In this case, the pressure $p_{\nu}$ is given by the equation

$$
\Delta p_{\nu}=-\operatorname{div}\left(v_{\nu} \cdot \nabla v_{\nu}\right)
$$

The mathematical theory of the Navier-Stokes equations (1.2) was initiated by Leray in [18]. He proved the global existence of a weak solution of the system (1.2) in the energy space by using a compactness method. Nevertheless, the uniqueness of these solutions is only known for two spatial dimensions. A few decades later, in [7], Fujita and Kato proved local well-posedness in the critical Sobolev space $\dot{H}^{1 / 2}\left(\mathbb{R}^{3}\right)$, by using a fixed point argument and taking advantage of the time decay of the heat semiflow. The global existence of these solutions is only proved for small initial data and the question for large data remains an outstanding open problem. For more discussion, we refer the reader, for example, to the papers [15], [16], and [22].

We can prove global existence when the initial data are not necessarily small but have some special symmetry. Before going further into the details, we first write the equation of the vorticity, which plays a central role in the theory of the global well-posedness. For a given vector field $v$, the vorticity $\omega$ is defined by $\omega=\operatorname{curl} v=\nabla \times v$, and, in the case of the system (1.2), it solves the transportdiffusion equation

$$
\partial_{t} \omega_{\nu}+v_{\nu} \cdot \nabla \omega_{\nu}-\nu \Delta \omega_{\nu}=\omega_{\nu} \cdot \nabla v_{\nu}
$$

The main difficulty is related to the dynamics of the stretching term $\omega_{\nu} \cdot \nabla v_{\nu}$. Now we will see how to use the axisymmetry of the flows in order to simplify the stretching term. We start with the following definition.

Definition 1.1. We say that a vector field $v$ is axisymmetric (without swirl) if it takes the form:

$$
v(t, x)=v^{r}(t, r, z) e_{r}+v^{z}(t, r, z) e_{z},
$$

where $z=x_{3}, x=\left(x_{1}, x_{2}, z\right), r=\left(x_{1}^{2}+x_{2}^{2}\right)^{1 / 2}$, and $\left(e_{r}, e_{\theta}, e_{z}\right)$ is the cylindrical basis of $\mathbb{R}^{3}$, given by

$$
e_{r}=\left(\frac{x_{1}}{r}, \frac{x_{2}}{r}, 0\right), \quad e_{\theta}=\left(-\frac{x_{2}}{r}, \frac{x_{1}}{r}, 0\right), \quad \text { and } \quad e_{z}=(0,0,1) .
$$

The components $v^{r}$ and $v^{z}$ do not depend on the angular variable $\theta$.

In what follows we need to recall some basic algebraic properties related to some computations in the cylindrical coordinate system. For example, for an axisymmetric vector field $v$, the operators $v \cdot \nabla$ and div have the forms

$$
v \cdot \nabla=v^{r} \partial_{r}+\frac{1}{r} v^{\theta} \partial_{\theta}+v^{z} \partial_{z}=v^{r} \partial_{r}+v^{z} \partial_{z}
$$


and

$$
\operatorname{div} v=\partial_{r} v^{r}+\frac{v^{r}}{r}+\partial_{z} v^{z}
$$

The vorticity $\omega$ of the vector field $v$ has the special form

$$
\omega=\left(\partial_{z} v^{r}-\partial_{r} v^{z}\right) e_{\theta}:=\omega^{\theta} e_{\theta}
$$

and the stretching term reads

$$
\omega \cdot \nabla v=\frac{v^{r}}{r} \omega
$$

Consequently, the equation (1.3) becomes

$$
\partial_{t} \omega_{\nu}+v_{\nu} \cdot \nabla \omega_{\nu}-\nu \Delta \omega_{\nu}=\frac{v_{\nu}^{r}}{r} \omega_{\nu}
$$

The expression of the Laplacian operator in cylindrical coordinates is given by $\Delta=\partial_{r r}+\frac{1}{r} \partial_{r}+\partial_{z z}$. Therefore, the scalar component $\omega_{\nu}^{\theta}$ of the vorticity will satisfies the equation

$$
\partial_{t} \omega_{\nu}^{\theta}+v_{\nu} \cdot \nabla \omega_{\nu}^{\theta}-\nu\left(\Delta \omega_{\nu}^{\theta}-\frac{\omega_{\nu}^{\theta}}{r^{2}}\right)=\frac{v_{\nu}^{r}}{r} \omega_{\nu}^{\theta}
$$

We can easily check that the quantity $\beta:=\omega_{\nu}^{\theta} / r$ solves the equation

$$
\partial_{t} \beta+v \cdot \nabla \beta-\nu\left(\Delta+\frac{2}{r} \partial_{r}\right) \beta=0 \text {. }
$$

Hence, we deduce that for all $p \in[1, \infty]$,

$$
\|\beta(t)\|_{L^{p}} \leq\left\|\beta^{0}\right\|_{L^{p}} .
$$

These new conservation laws enabled Ukhoviskii and Iudovich [25], to show the global well-posedness under the assumption $v^{0} \in H^{1}$ and $\omega_{0}, \omega_{0} / r \in L^{2} \cap L^{\infty}$. This result has been recently improved by many authors in various function spaces. The inviscid case $\nu=0$ has also been treated. For more details see for example [1], [2], [6], [14], [17], and [23].

Concerning the inviscid limit problem, that is the convergence of the viscous solutions $\left(v_{\nu}\right)_{\nu>0}$ to the solution of the incompressible Euler equation, we will restrict ourselves to the discussion of the following results. In [19], Majda proved that for $v^{0} \in H^{s}$ with $s>5 / 2$, the solutions $\left(v_{\nu}\right)_{\nu>0}$ converge in $L^{2}$ norm to the unique solution $v$ of the Euler system and the rate of convergence is of order $\nu t$. By using an elementary interpolation argument we deduce strong convergence in the Sobolev spaces $H^{\eta}$, for all $\eta<s$. We note that this result is local in time in space dimension 3 and global in space dimension 2. Recently, Masmoudi proved in [20] strong convergence for the same space $H^{s}$ of initial data; his proof is based on the use of a cutoff procedure. We mention that the inviscid limit problem in the context of axisymmetric flows was studied in [14].

Next we discuss the stratified Navier-Stokes system (1.1), which has been intensively studied in recent decades, and for which there have been proved many results related to the global well-posedness problem. 
The study of the case of stratified Euler equations with axisymmetric initial data was initiated by Hmidi and Rousset in [12]. This system is described by

$$
\left\{\begin{array}{l}
\partial_{t} v+v \cdot \nabla v+\nabla p=\rho e_{z} \\
\partial_{t} \rho+v \cdot \nabla \rho-\Delta \rho=0 \\
\operatorname{div} v=0 \\
v_{\mid t=0}=v^{0}, \quad \rho_{\mid t=0}=\rho^{0} .
\end{array}\right.
$$

To show global existence, one needs new a priori estimates, especially for the function $\zeta:=\omega_{\theta} / r$, which solves the equation

$$
\partial_{t} \zeta+v \cdot \nabla \zeta=-\frac{\partial_{r} \rho}{r} .
$$

The main difficulty is to find a priori estimates on the density $\rho$ to control the righthand side of the last equation. The idea is that the singularity $1 / r$ on the axis $r=0$ is a derivative and that the term $\partial_{r} \rho / r$ can be thought of as the Laplacian of the density $\rho$. In [12], the authors try to use smoothing effects to control this term $\partial_{r} \rho / r$. They gave a positive answer under the assumptions

$$
v^{0} \in H^{s}, \quad \rho^{0} \in H^{s-2} \cap L^{m}, s>5 / 2, m>6 \quad \text { and } \quad r^{2} \rho^{0} \in L^{2} .
$$

Their basic idea consists in using the coupled function $\Gamma:=\zeta+\frac{\partial_{r}}{r} \Delta^{-1} \rho$, which satisfies the transport equation

$$
\partial_{t} \Gamma+v \cdot \nabla \Gamma=-\left[\frac{\partial_{r}}{r} \Delta^{-1}, v \cdot \nabla\right] \rho .
$$

Since the operator $\frac{\partial_{r}}{r} \Delta^{-1}$ behaves like the Riesz transform on the class of axisymmetric functions, the estimate of $\|\zeta(t)\|_{L^{3,1}}$ is equivalent to a bound on $\|\Gamma(t)\|_{L^{3,1} \text {. }}$ Therefore the difficulty reduces to estimating the singular commutator which arises in the equation of $\Gamma$. For this purpose the authors used intensively the axisymmetric structure of the velocity combined with some tools of harmonic analysis and paradifferential calculus. The result of [12] is extended in [24] to the framework of critical Besov spaces. More precisely, global existence was proved for

$$
v^{0} \in B_{2,1}^{5 / 2}, \quad \rho^{0} \in B_{2,1}^{1 / 2} \cap L^{m}, m>6 \quad \text { and } \quad r^{2} \rho^{0} \in L^{2} .
$$

The aim of this paper is twofold. First, we extend the result of [12] to the stratified Navier-Stokes system (1.1) with uniform bounds with respect to the viscosity but for the subcritical regularities, that is, $\left(v^{0}, \rho^{0}\right) \in H^{s} \times H^{s-2}$ with $s>5 / 2$. Second, we analyze the inviscid limit problem and we show strong convergence of the solutions $\left(v_{\nu}, \rho_{\nu}\right)$ of the system (1.1) to the one of (1.6) in the same space of initial data. We point out that our approach for the last point is completely different from that in Masmoudi's work [20] for the incompressible Navier-Stokes equation.

First, we introduce the following space:

$$
u \in \chi_{m}^{s} \Longleftrightarrow u \in H^{s-2} \cap L^{m} \text { and such that } r^{2} u \in L^{2} .
$$

We state now our main result. 
Theorem 1.2. Let $s>5 / 2, v^{0} \in H^{s}$ be an axisymmetric divergence-free vector field without swirl, and let $\rho^{0} \in \chi_{m}^{s}$ with $m>6$ be an axisymmetric function. Then there exists a unique global solution $\left(v_{\nu}, \rho_{\nu}\right)$ of the system (1.1) such that

$$
v_{\nu} \in \mathcal{C}\left(\mathbb{R}_{+} ; H^{s}\right) \quad \text { and } \quad \rho_{\nu} \in \mathcal{C}\left(\mathbb{R}_{+} ; \chi_{m}^{s}\right) \cap L_{\text {loc }}^{1}\left(\mathbb{R}_{+} ; \text {Lip }\right),
$$

with uniform bounds with respect to the viscosity.

Moreover, for any $T>0$ we have

$$
\lim _{\nu \rightarrow 0}\left\|\left(v_{\nu}-v, \rho_{\nu}-\rho\right)\right\|_{L_{T}^{\infty}\left(H^{s} \times H^{s-2}\right)}=0,
$$

where $(v, \rho)$ is the solution of the system (1.6) with initial data $\left(v^{0}, \rho^{0}\right)$.

Before giving some details about the proof, a few remarks are in order.

Remarks. (1) From the proof the rate of convergence in the $L^{2}$ space is of order $\nu t$. More precisely,

$$
\left\|\left(v_{\nu}-v, \rho_{\nu}-\rho\right)(t)\right\|_{L^{2}} \leq \nu t f(t),
$$

with $f$ is an explicit function depending only on the size of the initial data and the variable time $t$.

(2) Our approach does not permit to treat the critical case $v_{0} \in B_{2,1}^{5 / 2}, \rho_{0} \in B_{2,1}^{1 / 2}$. Even though, we can extend the result of Proposition 3.2 to the Lorentz space $L^{3,1}$, the difficulty relies on the establishment of maximal smoothing effects for a transport-diffusion model in Lorentz space.

Now, we will discuss the main ideas of the proof of Theorem 1.2. To simplify the notation, we will write $(v, \rho)$ instead of $\left(v_{\nu}, \rho_{\nu}\right)$. First, recall that the vorticity $\omega=\omega^{\theta} e_{\theta}$ satisfies

$$
\partial_{t} \omega+v \cdot \nabla \omega-\nu \Delta \omega=\frac{v^{r}}{r} \omega+\operatorname{curl}\left(\rho e_{z}\right)
$$

This yields

$$
\partial_{t} \omega_{\theta}+v \cdot \nabla \omega_{\theta}-\nu\left(\Delta \omega_{\theta}-\frac{\omega_{\theta}}{r^{2}}\right)=\frac{v^{r}}{r} \omega_{\theta}-\partial_{r} \rho
$$

It follows that $\zeta:=\omega_{\theta} / r$ obeys the equation

$$
\partial_{t} \zeta+v \cdot \nabla \zeta-\nu\left(\Delta+\frac{2}{r} \partial_{r}\right) \zeta=-\frac{\partial_{r} \rho}{r} .
$$

At this stage, we can try to use the method of [12], but unfortunately it seems to be rigid and fails for the viscous case. Our alternative approach relies on the use of maximal smoothing effects combined with a suitable commutator estimate.

To be more precise, we use an interpolation argument combined with the maximum principle to obtain, for $\bar{p}>3$,

$$
\|\zeta(t)\|_{L^{3,1}} \leq C\|\zeta(t)\|_{L^{2} \cap L^{\bar{p}}} \leq C\left\|\zeta^{0}\right\|_{L^{2} \cap L^{\bar{p}}}+C \int_{0}^{t}\left\|\frac{\partial_{r} \rho}{r}(\tau)\right\|_{L^{2} \cap L^{\bar{p}}} d \tau .
$$


As we will see the restriction of the operator $\partial_{r} / r$ to the class of axisymmetric functions is dominated by the second derivative:

$$
\left\|\frac{\partial_{r}}{r} \rho(t)\right\|_{L^{p}} \leq C\left\|\nabla^{2} \rho(t)\right\|_{L^{p}}
$$

To estimate this last latter quantity we use the maximal smoothing effect of the heat flow and the difficulty reduces to the analysis of the commutator $\sum_{j}\left\|\left[\Delta_{j}, v \cdot \nabla\right] \rho\right\|_{L^{p}}$ which is the hard technical part of this paper. We shall prove in Proposition 3.2 that, for $p \in(1,+\infty)$,

$$
\begin{aligned}
& \sum_{j \geq-1}\left\|\left[\Delta_{j}, v \cdot \nabla\right] \rho\right\|_{L^{p}} \\
& \quad \leq C\|v\|_{L^{2}}\|\rho\|_{L^{p}}+C\left\|\frac{\omega_{\theta}}{r}\right\|_{L^{3,1} \cap L^{p}}\left(\left\|x_{h} \rho\right\|_{B_{\infty, 1}^{0}}+\|\rho\|_{B_{p, 1}^{0} \cap L^{\infty}}\right),
\end{aligned}
$$

where $x_{h}:=\left(x_{1}, x_{2}\right)$. Consequently, we obtain

$$
\|\zeta(t)\|_{L^{2} \cap L^{\bar{p}}} \leq C(t) e^{C\left\|x_{h} \rho\right\|_{L_{t}^{1} B_{\infty, 1}^{0}}}
$$

To estimate $\left\|x_{h} \rho\right\|_{L_{t}^{1} B_{\infty, 1}^{0}}$, we use the following inequality proved in [12]:

$$
\left\|x_{h} \rho\right\|_{L_{t}^{1} B_{\infty, 1}^{0}} \leq C_{0}(t)\left(1+\int_{0}^{t} h(\tau) \log \left(2+\|\zeta\|_{L_{\tau}^{\infty} L^{3,1}}\right) d \tau\right)
$$

where $t \mapsto C_{0}(t)$ is a given continuous function and $t \mapsto h(t)$ belongs to $L_{\text {loc }}^{1}\left(\mathbb{R}_{+}\right)$. We conclude by using (1.8) and (1.9) combined with the Gronwall inequality to obtain a global bound for $\|\zeta(t)\|_{L^{3,1}}$, uniformly with respect to the viscosity.

Concerning the inviscid limit, we first prove strong convergence in $L_{\text {loc }}^{\infty}\left(\mathbb{R}_{+} ; L^{2}\right)$ by making energy estimates. However the strong convergence in the space for initial data in $H^{s} \times H^{s-2}$ is more subtle. For this purpose we use interpolation arguments combined with an additional frequency decay of the energy uniformly with respect to $t$ and $\nu$ in the spirit of [9] and [13].

This paper is organized as follows. In Section 2, we fix some notation, give the definition of Besov and Lorentz spaces, and state some smoothing effects for a transport-diffusion equation. In Section 3, we study the estimate of the commutator $\sum_{j \geq-1}\left[\Delta_{j}, v \cdot \nabla\right] \rho$ in $L^{p}$ spaces. In the last section, we give the proof of Theorem 1.2, which will be presented in several steps.

\section{Tools and functional spaces}

In this preliminary section, we introduce some basic notations and recall the definitions of the usual and heterogeneous Besov spaces. We give also some results about Lorentz spaces and discuss some well-known results about the Littlewood-Paley decomposition and a transport-diffusion equation used later. 


\subsection{Notation}

For any positive $A$ and $B$, the notation $A \lesssim B$ means that there exists a positive constant $C$ independent of $A$ and $B$ and such that $A \leqslant C B$.

For any pair of operators $X$ and $Y$ acting on some Banach space $\mathcal{A}$, the commutator $[X, Y]$ is defined by $X Y-Y X$.

For $l \in \mathbb{N}$, we set

$$
\Phi_{l}(t)=C_{0} \underbrace{\exp (\ldots \exp }_{l \text { times }}\left(C_{0} t^{19 / 6}\right) \ldots),
$$

where $C_{0}$ depends on the norms of the initial data and its value may vary from line to line up to some absolute constants, but it does not depend on the viscosity $\nu$. We will make intensive use of the trivial facts

$$
\int_{0}^{t} \Phi_{l}(\tau) d \tau \leq \Phi_{l}(t) \text { and } \exp \left(\int_{0}^{t} \Phi_{l}(\tau) d \tau\right) \leq \Phi_{l+1}(t) .
$$

To define Besov spaces we need the following dyadic partition of unity (see [5], [16]).

Proposition 2.1. There exist two nonnegative radial functions $\chi \in \mathbb{C}_{0}^{\infty}\left(\mathbb{R}^{3}\right)$ and $\varphi \in \mathbb{C}_{0}^{\infty}\left(\mathbb{R}^{3} \backslash\{0\}\right)$ such that

$$
\begin{gathered}
\chi(\xi)+\sum_{j \geq 0} \varphi\left(2^{-j} \xi\right)=1, \quad \forall \xi \in \mathbb{R}^{3}, \\
|p-j| \geq 2 \Rightarrow \operatorname{supp} \varphi\left(2^{-p} \cdot\right) \cap \operatorname{supp} \varphi\left(2^{-j} \cdot\right)=\varnothing, \\
j \geq 1 \Rightarrow \operatorname{supp} \chi \cap \operatorname{supp} \varphi\left(2^{-j} \cdot\right)=\varnothing .
\end{gathered}
$$

Let $f \in \mathcal{S}^{\prime}\left(\mathbb{R}^{3}\right)$. We define the nonhomogeneous Littlewood-Paley operators by

$$
\Delta_{-1} f=\chi(\mathrm{D}) f, \quad \forall j \geq 0, \quad \Delta_{j} f=\varphi\left(2^{-j} \mathrm{D}\right) f \text { and } S_{j} f=\sum_{-1 \leq k \leq j-1} \Delta_{k} f .
$$

It may easily be checked that

$$
f=\sum_{j \geq-1} \Delta_{j} f, \quad \forall f \in \mathcal{S}^{\prime}\left(\mathbb{R}^{3}\right) .
$$

Moreover, the Littlewood-Paley operators satisfy the property of almost orthogonality: for any $f, g \in \mathcal{S}^{\prime}\left(\mathbb{R}^{3}\right)$,

$$
\Delta_{p} \Delta_{j} f=0 \quad \text { if }|p-j| \geqslant 2 ; \quad \Delta_{p}\left(S_{j-1} f \Delta_{j} g\right)=0 \quad \text { if }|p-j| \geqslant 5 .
$$

The following Bernstein inequality will be used constantly in the paper; see [5].

Lemma 2.2. There exists a constant $C>0$ such that for every $j, k \in \mathbb{N}$ and for every function $v$ we have

$$
\begin{aligned}
& \sup _{|\alpha|=k}\left\|\partial^{\alpha} S_{j} v\right\|_{L^{p_{2}}} \leq C^{k} 2^{j\left(k+3\left(1 / p_{1}-1 / p_{2}\right)\right)}\left\|S_{j} v\right\|_{L^{p_{1}}}, \text { for } p_{2} \geq p_{1} \geq 1, \\
& C^{-k} 2^{j k}\left\|\Delta_{j} v\right\|_{L^{p_{1}}} \leq \sup _{|\alpha|=k}\left\|\partial^{\alpha} \Delta_{j} v\right\|_{L^{p_{1}}} \leq C^{k} 2^{j k}\left\|\Delta_{j} v\right\|_{L^{p_{1}}} .
\end{aligned}
$$


From the paradifferential calculus introduced by J.-M. Bony in [4], the product $u v$ can be formally divided into three parts as follows:

$$
f g=T_{f} g+T_{g} f+R(f, g),
$$

where

$$
T_{f} g \stackrel{\text { def }}{=} \sum_{j} S_{j-1} f \Delta_{j} g
$$

and

$$
R(f, g)=\sum_{j} \Delta_{j} f \widetilde{\Delta}_{j} g, \text { with } \quad \widetilde{\Delta}_{j}=\Delta_{j-1}+\Delta_{j}+\Delta_{j+1}
$$

\subsection{Usual and heterogeneous Besov spaces}

We recall now the following definition of general Besov spaces.

Definition 2.3. Let $s \in \mathbb{R}$ and $1 \leq p, r \leq+\infty$. The inhomogeneous Besov space $B_{p, r}^{s}$ is the set of tempered distributions $f$ such that

$$
\|f\|_{B_{p, r}^{s}}:=\left(2^{j s}\left\|\Delta_{j} f\right\|_{L^{p}}\right)_{\ell^{r}}<+\infty .
$$

The following embeddings are an easy consequence of the Bernstein inequalities,

$$
B_{p_{1}, r_{1}}^{s} \hookrightarrow B_{p_{2}, r_{2}}^{s+3\left(1 / p_{2}-1 / p_{1}\right)}, \quad p_{1} \leq p_{2} \text { and } r_{1} \leq r_{2} .
$$

Let $T>0, \rho \geq 1,(p, r) \in[1, \infty]^{2}$, and $s \in \mathbb{R}$. We denote by $L_{T}^{\rho} B_{p, r}^{s}$ the space of distributions $f$ such that

$$
\|f\|_{L_{T}^{\rho} B_{p, r}^{s}}:=\left\|\left(2^{j s}\left\|\Delta_{j} f\right\|_{L^{p}}\right)_{\ell^{r}}\right\|_{L_{T}^{\rho}}<+\infty .
$$

We say that $f$ belongs to the Chemin-Lerner space $\widetilde{L}_{T}^{\rho} B_{p, r}^{s}$ if

$$
\|f\|_{\widetilde{L}_{T}^{\rho} B_{p, r}^{s}}:=\left\|2^{j s}\right\| \Delta_{j} f\left\|_{L_{T}^{\rho} L^{p}}\right\|_{\ell^{r}}<+\infty .
$$

The relations between these spaces are detailed in the following lemma, which is a direct consequence of the Minkowski inequality.

Lemma 2.4. Let $s \in \mathbb{R}, \varepsilon>0$, and $(p, r, \rho) \in[1,+\infty]^{3}$. Then we have the embeddings

$$
\begin{gathered}
L_{T}^{\rho} B_{p, r}^{s} \hookrightarrow \widetilde{L}_{T}^{\rho} B_{p, r}^{s} \hookrightarrow L_{T}^{\rho} B_{p, r}^{s-\varepsilon} \quad \text { if } r \geqslant \rho, \\
L_{T}^{\rho} B_{p, r}^{s+\varepsilon} \hookrightarrow \widetilde{L}_{T}^{\rho} B_{p, r}^{s} \hookrightarrow L_{T}^{\rho} B_{p, r}^{s} \quad \text { if } \rho \geq r .
\end{gathered}
$$

We remark that the Sobolev space $H^{s}$ coincides with the Besov space $B_{2,2}^{s}$ for $s \in \mathbb{R}$ and we have the embedding

$$
H^{s} \hookrightarrow L^{p}, \forall 0 \leq s<\frac{d}{2}, \quad \text { with } p=\frac{2 d}{d-2 s} .
$$

Now we will introduce the heterogeneous Besov spaces, which are extensions of the classical Besov spaces. 
Definition 2.5. (i) A function $\Psi:\{-1\} \cup \mathbb{N} \rightarrow \mathbb{R}_{+}^{*}$ belongs to the class $\mathcal{U}$ if the following conditions are satisfied:

(a) $\Psi$ is a nondecreasing function.

(b) There exists $C>0$ such that

$$
\sup _{x \in \mathbb{N} \cup\{-1\}} \frac{\Psi(x+1)}{\Psi(x)} \leq C .
$$

(ii) The class $\mathcal{U}_{\infty}$ is the set of functions $\Psi \in \mathcal{U}$ satisfying $\lim _{x \rightarrow+\infty} \Psi(x)=+\infty$.

(iii) Let $s \in \mathbb{R},(p, r) \in[1, \infty]^{2}$, and $\Psi \in \mathcal{U}$. We define the heterogeneous Besov space $B_{p, r}^{s, \Psi}$ as follows:

$$
u \in B_{p, r}^{s, \Psi} \text { if and only if }\|u\|_{B_{p, r}^{s, \Psi}}:=\left(\Psi(q) 2^{q s}\left\|\Delta_{q} u\right\|_{L^{p}}\right)_{\ell^{r}}<+\infty .
$$

Observe that when the profile $\Psi$ has exponential growth, $\Psi(q)=2^{\alpha q}, \alpha \in \mathbb{R}_{+}$, then the heterogeneous Besov space $B_{p, r}^{s, \Psi}$ reduces to the classical Besov space $B_{p, r}^{s+\alpha}$. When the profile $\Psi$ is a nonnegative constant, it is clear that $B_{p, r}^{s, \Psi}=B_{p, r}^{s}$.

The next result shows that any element of a given Besov space is always more regular than the prescribed regularity (see [9] for a proof).

Lemma 2.6. Let $s \in \mathbb{R}, p \in[1,+\infty], r \in[1,+\infty)$, and $f \in B_{p, r}^{s}$. Then there exists a function $\Psi \in \mathcal{U}_{\infty}$ such that $f \in B_{p, r}^{s, \Psi}$.

The following proposition will be useful later; see [11] for a proof.

Proposition 2.7. We have the following estimates:

a) Let $p \in[1, \infty]$, and let $f, g$ and $h$ be three functions such that $x h \in L^{1}$, $\nabla f \in L^{p}$, and $g \in L^{\infty}$. Then

$$
\|h *(f g)-f(h * g)\|_{L^{p}} \leq\|x h\|_{L^{1}}\|\nabla f\|_{L^{p}}\|g\|_{L^{\infty}} .
$$

b) Assume that $x h \in L^{1}, \nabla f \in L^{\infty}$, and $g \in L^{p}, \forall p \in[1, \infty]$. Then we have

$$
\|h *(f g)-f(h * g)\|_{L^{p}} \leq\|x h\|_{L^{1}}\|\nabla f\|_{L^{\infty}}\|g\|_{L^{p}} .
$$

\subsection{Lorentz spaces and interpolation}

Before defining the Lorentz spaces, we recall the notation of a nonincreasing rearrangement. The nonincreasing rearrangement $h^{*}: \mathbb{R}_{+} \rightarrow \mathbb{R}_{+}$of a measurable function $h$ is defined by the formula

$$
h^{*}(t):=\inf \left\{s^{\prime} \geq 0: l\left(\left\{y,|h(y)|>s^{\prime}\right\}\right) \leq t\right\},
$$

where $l$ denotes the usual Lebesgue measure. 
Definition 2.8 (Lorentz space). Let $h$ a measurable function and $1 \leq p \leq \infty$. Then $h$ belongs to the Lorentz space if

$$
\|h\|_{L^{p, r}}:=\left\{\begin{array}{l}
\left(\int_{0}^{\infty}\left(t^{1 / p} h^{*}(t)\right)^{r} \frac{d t}{t}\right)^{1 / r}<\infty \text { if } 1 \leq r<\infty \\
\sup _{t>0} t^{1 / p} h^{*}(t) \text { if } r=\infty .
\end{array}\right.
$$

We can also define the Lorentz spaces by interpolation between Lebesgue spaces,

$$
\left(L^{p_{1}}, L^{p_{2}}\right)_{(\mu, r)}=L^{p, r},
$$

where $1 \leq p_{1}<p<p_{2} \leq \infty, 1 / p=(1-\mu) / p_{1}+\mu / p_{2}$, and $1 \leq r \leq \infty$.

We have the classical properties:

$$
\begin{gathered}
\|u v\|_{L^{p, r}} \leq C\|u\|_{L^{\infty}}\|v\|_{L^{p, r}} \\
L^{p, r} \hookrightarrow L^{p, r_{1}}, \forall 1 \leq p \leq \infty, 1 \leq r \leq r_{1} \leq \infty, \quad \text { and } \quad L^{p, p}=L^{p} .
\end{gathered}
$$

We have also $L^{3,1}=\left(L^{2}, L^{\bar{p}}\right)_{(\mu, 1)}$ with $3<\bar{p}$ and we deduce that

$$
\|u\|_{L^{3,1}} \leq C\|u\|_{L^{2} \cap L^{\bar{p}}} \quad \text { with } 3<\bar{p} .
$$

The following lemma will be used later; see for instance [16] and [21].

Lemma 2.9. There exists a constant $C>0$ such that, for every $0<\beta<3$,

$$
\|f * g\|_{L^{\infty}\left(\mathbb{R}^{3}\right)} \leq C\|f\|_{L^{3 / \beta, \infty}\left(\mathbb{R}^{3}\right)}\|g\|_{L^{3 /(3-\beta), 1}\left(\mathbb{R}^{3}\right)} .
$$

Using Lemma 2.9 and the fact that $1 /|x|^{2} \in L^{3 / 2, \infty}\left(\mathbb{R}^{3}\right)$, we get

$$
\left\|\nabla \Delta^{-1} f\right\|_{L^{\infty}\left(\mathbb{R}^{3}\right)} \lesssim\left\|\frac{1}{|x|^{2}}\right\|_{L^{3 / 2, \infty}\left(\mathbb{R}^{3}\right)}\|f\|_{L^{3,1}\left(\mathbb{R}^{3}\right)} \lesssim\|f\|_{L^{3,1}\left(\mathbb{R}^{3}\right)} .
$$

\subsection{Estimates for a transport-diffusion equation}

We will give now some useful estimates for any smooth solution of the linear transport-diffusion model given by

$$
\left\{\begin{aligned}
\partial_{t} f+v \cdot \nabla f-\kappa \Delta f & =g \\
f_{\mid t=0} & =f^{0} .
\end{aligned}\right.
$$

We will give estimates of two kinds. The first are $L^{p}$ estimates, and the second concern the smoothing effects. We start with the $L^{p}$ estimates; see [8].

Lemma 2.10. Let $v$ be a smooth divergence-free vector field on $\mathbb{R}^{3}$ and let $f$ be a smooth solution of $(2.5)$. Then for all $p \in[1, \infty]$ and for every $\kappa \geq 0$, we have

$$
\|f(t)\|_{L^{p}} \leq\left\|f^{0}\right\|_{L^{p}}+\int_{0}^{t}\|g(\tau)\|_{L^{p}} d \tau .
$$


We need the following result; see [10] for a proof.

Proposition 2.11. Let $v$ be a smooth divergence-free vector field on $\mathbb{R}^{3}$ with vorticity $\omega:=\operatorname{curl} v$. Let $f$ be a smooth solution of (2.5) with $\kappa=1$ and $g=0$. Then, for every $j \in \mathbb{N}$, and $f^{0} \in L^{p}$ with $1 \leq p \leq \infty$ and $t \geq 0$, we have

$$
2^{2 j}\left\|\Delta_{j} f\right\|_{L_{t}^{1} L^{p}} \lesssim\left\|f^{0}\right\|_{L^{p}}\left(1+(j+1)\|\omega\|_{L_{t}^{1} L^{\infty}}+\left\|\nabla \Delta_{-1} v\right\|_{L_{t}^{1} L^{\infty}}\right) .
$$

We will need the following smoothing effects which are proved in [12].

Proposition 2.12. Let $v$ be a smooth divergence-free vector field on $\mathbb{R}^{3}$ and let $f$ be a smooth solution of (2.5) with $\kappa=1$. Then, for every $j \in \mathbb{N}, p \geq 2$, and $t \geq 0$, we have

$$
\begin{aligned}
\left\|\Delta_{j} f\right\|_{L_{t}^{\infty} L^{p}}+2^{2 j} \int_{0}^{t}\left\|\Delta_{j} f(\tau)\right\|_{L^{p}} d \tau \lesssim & \left\|\Delta_{j} f^{0}\right\|_{L^{p}}+\int_{0}^{t}\left\|\left[\Delta_{j}, v \cdot \nabla\right] f(\tau)\right\|_{L^{p}} d \tau \\
& +\int_{0}^{t}\left\|\Delta_{j} g(\tau)\right\|_{L^{p}} d \tau
\end{aligned}
$$

\section{Commutator estimates}

In this section, we discuss the commutator of the operator $\Delta_{j}$ and the convection operator $v \cdot \nabla$. We start with the following estimate, which was proved in [9].

Proposition 3.1. Let $v$ be a smooth divergence-free vector field on $\mathbb{R}^{3}$ and let $u$ be a smooth function. Then for every $s>0, r \in[1,+\infty]$, and $\Psi \in \mathcal{U}$ as in Definition 2.5, we have the estimate

$$
\left(\Psi(j) 2^{j s}\left\|\left[\Delta_{j}, v \cdot \nabla\right] u\right\|_{L^{2}}\right)_{\ell^{r}} \lesssim\|\nabla v\|_{L^{\infty}}\|u\|_{B_{2, r}^{s, \Psi}}+\|\nabla u\|_{L^{\infty}}\|v\|_{B_{2, r}^{s, \Psi}} .
$$

We aim in this section to prove the following.

Proposition 3.2. Let $v$ be an axisymmetric smooth and divergence-free vector field without swirl and let $\rho$ be an axisymmetric smooth scalar function. Then for every $j \geq-1$ and $1<p<\infty$, we have the estimate

$$
\sum_{j \geq-1}\left\|\left[\Delta_{j}, v \cdot \nabla\right] \rho\right\|_{L^{p}} \lesssim\|v\|_{L^{2}}\|\rho\|_{L^{p}}+\left\|\frac{\omega_{\theta}}{r}\right\|_{L^{3,1} \cap L^{p}}\left(\left\|x_{h} \rho\right\|_{B_{\infty, 1}^{0}}+\|\rho\|_{B_{p, 1}^{0} \cap L^{\infty}}\right),
$$

where $\omega_{\theta}$ is the angular component of $\omega=\nabla \times v$.

Proof. First, using the decomposition (2.1) of Bony, we write

$$
\begin{aligned}
\sum_{j \geq-1}\left[\Delta_{j}, v \cdot \nabla\right] \rho= & \sum_{j \geq-1} \sum_{i=1}^{3}\left[\Delta_{j}, T_{v^{i}} \cdot\right] \partial_{i} \rho+\sum_{j \geq-1} \sum_{i=1}^{3}\left[\Delta_{j}, T_{\partial_{i}} \cdot v^{i}\right] \rho \\
& +\sum_{j \geq-1} \sum_{i=1}^{3}\left[\Delta_{j}, R\left(v^{i} \cdot, \partial_{i}\right)\right] \rho \\
:= & \mathrm{I}+\mathrm{II}+\mathrm{III} .
\end{aligned}
$$


Estimate of I. We start with the estimate of the first component I, that is, for $i=1$. Since $v$ is divergence-free, we have $\Delta v=-\nabla \times \omega$. Then for axisymmetric flows, we obtain that

$$
\begin{aligned}
v^{1}(x)=\Delta^{-1} \partial_{3} \omega^{2} & =\Delta^{-1} \partial_{3}\left(x_{1} \frac{\omega_{\theta}}{r}\right)=\Delta^{-1}\left(x_{1} \partial_{3} \frac{\omega_{\theta}}{r}\right) \\
& =x_{1} \Delta^{-1} \partial_{3}\left(\frac{\omega_{\theta}}{r}\right)-2 \partial_{13} \Delta^{-2}\left(\frac{\omega_{\theta}}{r}\right) .
\end{aligned}
$$

In the last line, we have used the following identity; see Lemma 2.10 in [12] for a proof.

Lemma 3.3. For every $f \in \mathcal{S}\left(\mathbb{R}^{3}, \mathbb{R}\right)$ and $i, j \in\{1,2,3\}$, we have

$$
\Delta^{-1}\left(x_{i} \partial_{j} f\right)=x_{i} \Delta^{-1} \partial_{j} f-2 \mathcal{R}_{i, j} \Delta^{-1} f,
$$

where $\mathcal{R}_{i, j}=\partial_{i, j} \Delta^{-1}$ is the Riesz transform.

Then we have

$$
\sum_{j}\left[\Delta_{j}, T_{v^{1}} \cdot\right] \partial_{1} \rho=\sum_{|q-j| \leq 4}\left[\Delta_{j}, S_{q-1} v^{1}\right] \Delta_{q} \partial_{1} \rho
$$$$
=\sum_{|q-j| \leq 4}\left[\Delta_{j}, S_{q-1}\left(x_{1} \Delta^{-1} \partial_{3}\left(\frac{\omega_{\theta}}{r}\right)\right)\right] \Delta_{q} \partial_{1} \rho-2 \sum_{|q-j| \leq 4}\left[\Delta_{j}, S_{q-1} \partial_{13} \Delta^{-2}\left(\frac{\omega_{\theta}}{r}\right)\right] \Delta_{q} \partial_{1} \rho .
$$

By the definition of $\Delta_{q}$, there exists a function $\varphi \in \mathcal{S}\left(\mathbb{R}^{3}\right)$ such that

$$
\begin{aligned}
x_{1} \Delta_{q} \rho & =x_{1} 2^{3 q} \int_{\mathbb{R}^{3}} \varphi\left(2^{q}(x-y)\right) \rho(y) d y \\
& =2^{3 q} \int_{\mathbb{R}^{3}} \varphi\left(2^{q}(x-y)\right) y_{1} \rho(y) d y+2^{3 q} \int_{\mathbb{R}^{3}} \varphi\left(2^{q}(x-y)\right)\left(x_{1}-y_{1}\right) \rho(y) d y \\
& =\Delta_{q}\left(x_{1} \rho\right)+2^{-q} 2^{3 q} \varphi_{1}\left(2^{q} \cdot\right) * \rho,
\end{aligned}
$$

where $\varphi_{1}(x)=x_{1} \varphi(x)$. Consequently the commutator reads,

$$
\left[\Delta_{q}, x_{1}\right] \rho=-2^{2 q} \varphi_{1}\left(2^{q} \cdot\right) * \rho .
$$

Similarly for the cutoff $S_{q}$, we obtain

$$
x_{1} S_{q} F=S_{q}\left(x_{1} F\right)+2^{2 q} \chi_{1}\left(2^{q} \cdot\right) * F,
$$

where $\chi_{1}(x)=x_{1} \chi(x) \in \mathcal{S}\left(\mathbb{R}^{3}\right)$.

To estimate the first term of (3.2), we use (3.4) to write

$$
\begin{aligned}
{\left[\Delta_{j},\right.} & \left.S_{q-1}\left(x_{1} \Delta^{-1} \partial_{3}\left(\frac{\omega_{\theta}}{r}\right)\right)\right] \Delta_{q} \partial_{1} \rho \\
= & {\left[\Delta_{j}, x_{1} S_{q-1}\left(\Delta^{-1} \partial_{3}\left(\frac{\omega_{\theta}}{r}\right)\right)\right] \Delta_{q} \partial_{1} \rho-\left[\Delta_{j}, 2^{2 q} \chi_{1}\left(2^{q} \cdot\right) * \Delta^{-1} \partial_{3}\left(\frac{\omega_{\theta}}{r}\right)\right] \Delta_{q} \partial_{1} \rho } \\
= & {\left[\Delta_{j}, S_{q-1}\left(\Delta^{-1} \partial_{3}\left(\frac{\omega_{\theta}}{r}\right)\right)\right] x_{1} \Delta_{q} \partial_{1} \rho+S_{q-1} \Delta^{-1} \partial_{3}\left(\frac{\omega_{\theta}}{r}\right)\left[\Delta_{j}, x_{1}\right] \Delta_{q} \partial_{1} \rho } \\
& -\left[\Delta_{j}, 2^{2 q} \chi_{1}\left(2^{q} \cdot\right) * \Delta^{-1} \partial_{3}\left(\frac{\omega_{\theta}}{r}\right)\right] \Delta_{q} \partial_{1} \rho .
\end{aligned}
$$


Therefore we obtain

$$
\begin{aligned}
& {\left[\Delta_{j}, S_{q-1}\left(x_{1} \Delta^{-1} \partial_{3}\left(\frac{\omega_{\theta}}{r}\right)\right)\right] \Delta_{q} \partial_{1} \rho} \\
& =\left[\Delta_{j}, S_{q-1}\left(\Delta^{-1} \partial_{3}\left(\frac{\omega_{\theta}}{r}\right)\right)\right] \partial_{1}\left(x_{1} \Delta_{q} \rho\right)-\left[\Delta_{j}, S_{q-1}\left(\Delta^{-1} \partial_{3}\left(\frac{\omega_{\theta}}{r}\right)\right)\right] \Delta_{q} \rho \\
& \quad+S_{q-1} \Delta^{-1} \partial_{3}\left(\frac{\omega_{\theta}}{r}\right)\left[\Delta_{j}, x_{1}\right] \Delta_{q} \partial_{1} \rho-\left[\Delta_{j}, 2^{2 q} \chi_{1}\left(2^{q} \cdot\right) * \Delta^{-1} \partial_{3}\left(\frac{\omega_{\theta}}{r}\right)\right] \Delta_{q} \partial_{1} \rho .
\end{aligned}
$$

Using (3.3) this gives

$$
\begin{aligned}
& {\left[\Delta_{j}, S_{q-1}\left(x_{1} \Delta^{-1} \partial_{3}\left(\frac{\omega_{\theta}}{r}\right)\right)\right] \Delta_{q} \partial_{1} \rho} \\
& =\left[\Delta_{j}, S_{q-1}\left(\Delta^{-1} \partial_{3}\left(\frac{\omega_{\theta}}{r}\right)\right)\right] \partial_{1} \Delta_{q}\left(x_{1} \rho\right)+\left[\Delta_{j}, S_{q-1}\left(\Delta^{-1} \partial_{3}\left(\frac{\omega_{\theta}}{r}\right)\right)\right] \partial_{1}\left(2^{2 q} \varphi_{1}\left(2^{q} \cdot\right) * \rho\right) \\
& \quad-\left[\Delta_{j}, S_{q-1}\left(\Delta^{-1} \partial_{3}\left(\frac{\omega_{\theta}}{r}\right)\right)\right] \Delta_{q} \rho S_{q-1} \Delta^{-1} \partial_{3}\left(\frac{\omega_{\theta}}{r}\right)\left(2^{2 j} \varphi_{1}\left(2^{j} \cdot\right) * \Delta_{q} \partial_{1} \rho\right) \\
& \quad-\left[\Delta_{j}, 2^{2 q} \chi_{1}\left(2^{q} \cdot\right) * \Delta^{-1} \partial_{3}\left(\frac{\omega_{\theta}}{r}\right)\right] \Delta_{q} \partial_{1} \rho .
\end{aligned}
$$

Therefore,

$$
\sum_{|q-j| \leq 4}\left[\Delta_{j}, S_{q-1}\left(x_{1} \Delta^{-1} \partial_{3}\left(\frac{\omega_{\theta}}{r}\right)\right)\right] \Delta_{q} \partial_{1} \rho:=\mathrm{I}_{1}+\mathrm{I}_{2}+\mathrm{I}_{3}+\mathrm{I}_{4}+\mathrm{I}_{5},
$$

where

$$
\begin{aligned}
& \mathrm{I}_{1}=\sum_{|q-j| \leq 4}\left[\Delta_{j}, S_{q-1}\left(\Delta^{-1} \partial_{3}\left(\frac{\omega_{\theta}}{r}\right)\right)\right] \partial_{1} \Delta_{q}\left(x_{1} \rho\right), \\
& \mathrm{I}_{2}=\sum_{|q-j| \leq 4}\left[\Delta_{j}, S_{q-1}\left(\Delta^{-1} \partial_{3}\left(\frac{\omega_{\theta}}{r}\right)\right)\right]\left(2^{3 q}\left(\partial_{1} \varphi_{1}\right)\left(2^{q} \cdot\right) * \rho\right), \\
& \mathrm{I}_{3}=-\sum_{|q-j| \leq 4}\left[\Delta_{j}, S_{q-1}\left(\Delta^{-1} \partial_{3}\left(\frac{\omega_{\theta}}{r}\right)\right)\right] \Delta_{q} \rho, \\
& \mathrm{I}_{4}=-\sum_{|q-j| \leq 4} S_{q-1} \Delta^{-1} \partial_{3}\left(\frac{\omega_{\theta}}{r}\right)\left(2^{2 j} \varphi_{1}\left(2^{j} \cdot\right) * \Delta_{q} \partial_{1} \rho\right) \\
& \mathrm{I}_{5}=-\sum_{|q-j| \leq 4}\left[\Delta_{j}, 2^{2 q} \chi_{1}\left(2^{q} \cdot\right) * \Delta^{-1} \partial_{3}\left(\frac{\omega_{\theta}}{r}\right)\right] \Delta_{q} \partial_{1} \rho .
\end{aligned}
$$

Estimate of $I_{1}$. We use Proposition 2.7-a), the continuity of the Riesz transform in the $L^{p}$, space and the Bernstein inequality:

$$
\begin{aligned}
\left\|\mathrm{I}_{1}\right\|_{L^{p}} & \leq \sum_{|q-j| \leq 4}\left\|x h_{j}\right\|_{L^{1}}\left\|\nabla S_{q-1} \Delta^{-1} \partial_{3}\left(\frac{\omega_{\theta}}{r}\right)\right\|_{L^{p}}\left\|\partial_{1} \Delta_{q}\left(x_{1} \rho\right)\right\|_{L^{\infty}} \\
& \lesssim \sum_{|q-j| \leq 4} 2^{-j}\|x h\|_{L^{1}}\left\|\nabla \Delta^{-1} \partial_{3}\left(\frac{\omega_{\theta}}{r}\right)\right\|_{L^{p}} 2^{q}\left\|\Delta_{q}\left(x_{1} \rho\right)\right\|_{L^{\infty}} \\
& \lesssim\left\|\frac{\omega_{\theta}}{r}\right\|_{L^{p}} \sum_{|q-j| \leq 4} 2^{q-j}\left\|\Delta_{q}\left(x_{1} \rho\right)\right\|_{L^{\infty}} \lesssim\left\|\frac{\omega_{\theta}}{r}\right\|_{L^{p}}\left\|x_{1} \rho\right\|_{B_{\infty}^{0}, 1}
\end{aligned}
$$

where $h_{j}(x)=2^{3 j} h\left(2^{j} x\right) \in \mathcal{S}\left(\mathbb{R}^{3}\right)$. 
Estimate of $\mathrm{I}_{2}$. Using Proposition 2.7-a), the continuity of the Riesz transform on Lebesgue space, and the Young inequalities for convolution, we get

$$
\begin{aligned}
\left\|\mathrm{I}_{2}\right\|_{L^{p}} & \leq \sum_{|q-j| \leq 4} 2^{-j}\|x h\|_{L^{1}}\left\|\nabla S_{q-1} \Delta^{-1} \partial_{3}\left(\frac{\omega_{\theta}}{r}\right)\right\|_{L^{p}}\left\|2^{3 q}\left(\partial_{1} \varphi_{1}\right)\left(2^{q} \cdot\right) * \rho\right\|_{L^{\infty}} \\
& \lesssim\left\|\nabla \Delta^{-1} \partial_{3}\left(\frac{\omega_{\theta}}{r}\right)\right\|_{L^{p}} \sum_{|q-j| \leq 4} 2^{-j} 2^{3 q}\left\|\left(\partial_{1} \varphi_{1}\right)\left(2^{q} \cdot\right)\right\|_{L^{1}}\|\rho\|_{L^{\infty}} \\
& \lesssim\left\|\frac{\omega_{\theta}}{r}\right\|_{L^{p}} \sum_{|q-j| \leq 4} 2^{q-j} 2^{-q}\left\|\partial_{1} \varphi_{1}\right\|_{L^{1}}\|\rho\|_{L^{\infty}} \lesssim\left\|\frac{\omega_{\theta}}{r}\right\|_{L^{p}}\|\rho\|_{L^{\infty}} .
\end{aligned}
$$

Estimate of $\mathrm{I}_{3}$. We use Proposition 2.7-a) and the continuity of the Riesz transform on the Lebesgue space:

$$
\begin{aligned}
\left\|\mathrm{I}_{3}\right\|_{L^{p}} & \lesssim \sum_{|q-j| \leq 4} 2^{-j}\|x h\|_{L^{1}}\left\|\nabla S_{q-1} \Delta^{-1} \partial_{3}\left(\frac{\omega_{\theta}}{r}\right)\right\|_{L^{p}}\left\|\Delta_{q} \rho\right\|_{L^{\infty}} \\
& \lesssim\left\|\frac{\omega_{\theta}}{r}\right\|_{L^{p}} \sum_{|q-j| \leq 4} 2^{-j}\left\|\Delta_{q} \rho\right\|_{L^{\infty}} \lesssim\left\|\frac{\omega_{\theta}}{r}\right\|_{L^{p}}\|\rho\|_{L^{\infty}} .
\end{aligned}
$$

Estimate of $\mathrm{I}_{4}$. Using now the Hölder inequality, the continuity of the operator $S_{q-1}$ in $L^{\infty}$ spaces, (2.4), the Young inequalities for convolution and the Bernstein inequality, we get

$$
\begin{aligned}
\left\|\mathrm{I}_{4}\right\|_{L^{p}} & \leq \sum_{|q-j| \leq 4}\left\|S_{q-1} \Delta^{-1} \partial_{3}\left(\frac{\omega_{\theta}}{r}\right)\right\|_{L^{\infty}}\left\|2^{2 j} \varphi_{1}\left(2^{j} \cdot\right) * \Delta_{q} \partial_{1} \rho\right\|_{L^{p}} \\
& \lesssim\left\|\frac{\omega_{\theta}}{r}\right\|_{L^{3,1}} \sum_{|q-j| \leq 4} 2^{q-j}\left\|\varphi_{1}\right\|_{L^{1}}\left\|\Delta_{q} \rho\right\|_{L^{p}} \lesssim\left\|\frac{\omega_{\theta}}{r}\right\|_{L^{3,1}}\|\rho\|_{B_{p, 1}^{0}} .
\end{aligned}
$$

Estimate of $I_{5}$. We use Proposition 2.7-a), the Young inequality for the convolutions, the continuity of the Riesz transform in $L^{p}$ spaces and the Bernstein inequality. We get

$$
\begin{aligned}
\left\|\mathrm{I}_{5}\right\|_{L^{p}} & \lesssim \sum_{|q-j| \leq 4} 2^{-j}\|x h\|_{L^{1}}\left\|2^{2 q} \chi_{1}\left(2^{q} \cdot\right) * \nabla \Delta^{-1} \partial_{3}\left(\frac{\omega_{\theta}}{r}\right)\right\|_{L^{p}}\left\|\Delta_{q} \partial_{1} \rho\right\|_{L^{\infty}} \\
& \lesssim \sum_{|q-j| \leq 4} 2^{-j} 2^{2 q}\left\|\chi_{1}\left(2^{q} \cdot\right)\right\| L_{L^{1}}\left\|\nabla \Delta^{-1} \partial_{3}\left(\frac{\omega_{\theta}}{r}\right)\right\|_{L^{p}} 2^{q}\left\|\Delta_{q} \rho\right\|_{L^{\infty}} \\
& \lesssim\left\|\frac{\omega_{\theta}}{r}\right\|_{L^{p}} \sum_{|q-j| \leq 4} 2^{-j} 2^{-q}\|\chi\|_{L^{1}} 2^{q}\left\|\Delta_{q} \rho\right\|_{L^{\infty}} \lesssim\left\|\frac{\omega_{\theta}}{r}\right\|_{L^{p}}\|\rho\|_{L^{\infty}}
\end{aligned}
$$

Finally, we obtain

$$
\begin{aligned}
\sum_{|q-j| \leq 4} \| & {\left[\Delta_{j}, S_{q-1}\left(x_{1} \Delta^{-1} \partial_{3}\left(\frac{\omega_{\theta}}{r}\right)\right)\right] \Delta_{q} \partial_{1} \rho \|_{L^{p}} } \\
& \lesssim\left\|\frac{\omega_{\theta}}{r}\right\|_{L^{3,1} \cap L^{p}}\left\|x_{1} \rho\right\|_{B_{\infty, 1}^{0}}+\left\|\frac{\omega_{\theta}}{r}\right\|_{L^{3,1} \cap L^{p}}\|\rho\|_{B_{p, 1}^{0} \cap L^{\infty}} .
\end{aligned}
$$


To estimate the second term of (3.2), we use Proposition 2.7-b), the Bernstein inequality, (2.4), and the continuity of the Riesz transform on the Lorentz spaces. We obtain:

$$
\begin{aligned}
\sum_{|q-j| \leq 4}\left\|\left[\Delta_{j}, S_{q-1} \partial_{13} \Delta^{-2}\left(\frac{\omega_{\theta}}{r}\right)\right] \Delta_{q} \partial_{1} \rho\right\|_{L^{p}} & \lesssim \sum_{|q-j| \leq 4}\left\|x h_{j}\right\|_{L^{1}}\left\|\nabla S_{q-1} \partial_{13} \Delta^{-2}\left(\frac{\omega_{\theta}}{r}\right)\right\|_{L^{\infty}}\left\|\Delta_{q} \partial_{1} \rho\right\|_{L^{p}} \\
(3.6) & \lesssim \sum_{|q-j| \leq 4} 2^{q-j}\|x h\|_{L^{1}}\left\|S_{q-1} \partial_{13} \Delta^{-1}\left(\frac{\omega_{\theta}}{r}\right)\right\|_{L^{3,1}}\left\|\Delta_{q} \rho\right\|_{L^{p}} \lesssim\left\|\frac{\omega_{\theta}}{r}\right\|_{L^{3,1}}\|\rho\|_{B_{p, 1}^{0}} .
\end{aligned}
$$

Plugging (3.5) and (3.6) into (3.2) we get

$$
\sum_{j}\left\|\left[\Delta_{j}, T_{v^{1}} \cdot\right] \partial_{1} \rho\right\|_{L^{p}} \lesssim\left\|\frac{\omega_{\theta}}{r}\right\|_{L^{3,1} \cap L^{p}}\left(\left\|x_{1} \rho\right\|_{B_{\infty, 1}^{0}}+\|\rho\|_{B_{p, 1}^{0} \cap L^{\infty}}\right) .
$$

The term $\sum_{j}\left[\Delta_{j}, T_{v^{2}} \cdot\right] \partial_{2} \rho$ can be estimated in the same way as above and we obtain the estimate

$$
\sum_{j}\left\|\left[\Delta_{j}, T_{v^{2}} \cdot\right] \partial_{2} \rho\right\|_{L^{p}} \lesssim\left\|\frac{\omega_{\theta}}{r}\right\|_{L^{3,1} \cap L^{p}}\left(\left\|x_{2} \rho\right\|_{B_{\infty, 1}^{0}}+\|\rho\|_{B_{p, 1}^{0} \cap L^{\infty}}\right) .
$$

The estimate of the term $\sum_{j}\left[\Delta_{j}, T_{v^{3}} \cdot\right] \partial_{3} \rho$ will be done as follows. Since we have

$$
\begin{aligned}
\Delta v^{3} & =-(\nabla \times \omega)_{3}=-\left(\partial_{r} \omega_{\theta}+\frac{\omega_{\theta}}{r}\right)=-\left(r \partial_{r}\left(\frac{\omega_{\theta}}{r}\right)+2 \frac{\omega_{\theta}}{r}\right) \\
& =-\left(x_{h} \cdot \nabla_{h}\left(\frac{\omega_{\theta}}{r}\right)+2 \frac{\omega_{\theta}}{r}\right),
\end{aligned}
$$

using Lemma 3.3, we get

$$
\begin{aligned}
-v^{3}(x) & =\Delta^{-1}\left(x_{h} \cdot \nabla_{h}\left(\frac{\omega_{\theta}}{r}\right)\right)+2 \Delta^{-1}\left(\frac{\omega_{\theta}}{r}\right) \\
& =x_{h} \cdot \Delta^{-1} \nabla_{h}\left(\frac{\omega_{\theta}}{r}\right)-2 \sum_{i=1}^{2} \partial_{i i} \Delta^{-2}\left(\frac{\omega_{\theta}}{r}\right)+2 \Delta^{-1}\left(\frac{\omega_{\theta}}{r}\right) \\
& =x_{h} \cdot \Delta^{-1} \nabla_{h}\left(\frac{\omega_{\theta}}{r}\right)+2 \partial_{33} \Delta^{-2}\left(\frac{\omega_{\theta}}{r}\right) .
\end{aligned}
$$

Then we have a decomposition of the commutator of the form,

$$
\begin{aligned}
-\sum_{j}\left[\Delta_{j}, T_{v^{3}} \cdot\right] \partial_{3} \rho= & \sum_{|q-j| \leq 4} \sum_{k=1}^{2}\left[\Delta_{j}, S_{q-1}\left(x_{k} \Delta^{-1} \partial_{k}\left(\frac{\omega_{\theta}}{r}\right)\right)\right] \Delta_{q} \partial_{3} \rho \\
& +2 \sum_{|q-j| \leq 4}\left[\Delta_{j}, S_{q-1} \partial_{33} \Delta^{-2}\left(\frac{\omega_{\theta}}{r}\right)\right] \Delta_{q} \partial_{3} \rho .
\end{aligned}
$$

This identity looks like (3.2), and by reproducing the analysis leading to (3.2), we get

$$
\sum_{j}\left\|\left[\Delta_{j}, T_{v^{3}} \cdot\right] \partial_{3} \rho\right\|_{L^{p}} \lesssim\left\|\frac{\omega_{\theta}}{r}\right\|_{L^{3,1} \cap L^{p}}\left(\sum_{k=1}^{2}\left\|x_{k} \rho\right\|_{B_{\infty, 1}^{0}}+\|\rho\|_{B_{p, 1}^{0} \cap L^{\infty}}\right) .
$$


Estimate of II. Let us now turn to the estimate of the second term II. We use (3.1), (3.3) and (3.4). With the same computations as for the term I, we get

$\sum_{j}\left[\Delta_{j}, T_{\partial_{1}} \cdot v^{1}\right] \rho=\sum_{|q-j| \leq 4}\left[\Delta_{j}, \Delta_{q} v^{1}\right] S_{q-1} \partial_{1} \rho:=\mathrm{II}_{1}+\mathrm{II}_{2}+\mathrm{II}_{3}+\mathrm{II}_{4}+\mathrm{II}_{5}+\mathrm{II}_{6}$,

where

$$
\begin{aligned}
& \mathrm{II}_{1}=\sum_{|q-j| \leq 4}\left[\Delta_{j}, \Delta_{q} \Delta^{-1} \partial_{3}\left(\frac{\omega_{\theta}}{r}\right)\right] \partial_{1} S_{q-1}\left(x_{1} \rho\right) \\
& \mathrm{II}_{2}=\sum_{|q-j| \leq 4}\left[\Delta_{j}, \Delta_{q} \Delta^{-1} \partial_{3}\left(\frac{\omega_{\theta}}{r}\right)\right]\left(2^{3 q}\left(\partial_{1} \chi_{1}\right)\left(2^{q} \cdot\right) * \rho\right) \\
& \mathrm{II}_{3}=-\sum_{|q-j| \leq 4}\left[\Delta_{j}, \Delta_{q} \Delta^{-1} \partial_{3}\left(\frac{\omega_{\theta}}{r}\right)\right] S_{q-1} \rho \\
& \mathrm{II}_{4}=-\sum_{|q-j| \leq 4} \Delta_{q} \Delta^{-1} \partial_{3}\left(\frac{\omega_{\theta}}{r}\right)\left(2^{2 j} \chi_{1}\left(2^{j} \cdot\right) * S_{q-1} \partial_{1} \rho\right) \\
& \mathrm{II}_{5}=-\sum_{|q-j| \leq 4}\left[\Delta_{j}, 2^{2 q} \varphi_{1}\left(2^{q} \cdot\right) * \Delta^{-1} \partial_{3}\left(\frac{\omega_{\theta}}{r}\right)\right] S_{q-1} \partial_{1} \rho \\
& \mathrm{II}_{6}=-2 \sum_{|q-j| \leq 4}\left[\Delta_{j}, \Delta_{q} \partial_{13} \Delta^{-2}\left(\frac{\omega_{\theta}}{r}\right)\right] S_{q-1} \partial_{1} \rho .
\end{aligned}
$$

To estimate $\mathrm{II}_{1}$, we do not need to use the structure of the commutator. We will use the Hölder and Bernstein inequalities and the following estimate: for every $p \in[1, \infty]$ we have that

$$
\left\|\Delta_{q} \Delta^{-1} \partial_{3} f\right\|_{L^{p}} \lesssim 2^{-q}\left\|\nabla \Delta_{q} \Delta^{-1} \partial_{3} f\right\|_{L^{p}} \lesssim 2^{-q}\|f\|_{L^{p}}, \quad \forall q \geq 0
$$

Thus we have

$$
\begin{aligned}
\left\|\mathrm{II}_{1}\right\|_{L^{p}} & \lesssim \sum_{|q-j| \leq 4}\left\|\Delta_{q}\left(\Delta^{-1} \partial_{3}\left(\frac{\omega_{\theta}}{r}\right)\right)\right\|_{L^{p}}\left\|\partial_{1} S_{q-1}\left(x_{1} \rho\right)\right\|_{L^{\infty}} \\
& \lesssim\left\|\frac{\omega_{\theta}}{r}\right\|_{L^{p}} \sum_{|q-j| \leq 4} 2^{-q} \sum_{-1 \leq k \leq q-2} 2^{k}\left\|\Delta_{k}\left(x_{1} \rho\right)\right\|_{L^{\infty}} \lesssim\left\|\frac{\omega_{\theta}}{r}\right\|_{L^{p}}\left\|x_{1} \rho\right\|_{B_{\infty, 1}^{0}} .
\end{aligned}
$$

The terms $\mathrm{II}_{2}, \mathrm{II}_{3}, \mathrm{II}_{4}, \mathrm{II}_{5}$ and $\mathrm{II}_{6}$ can be estimated in a manner similar to how $\mathrm{I}_{2}$, $\mathrm{I}_{3}, \mathrm{I}_{4}, \mathrm{I}_{5}$, and the second term of (3.2) were estimated. Finally, we conclude that

$$
\|\mathrm{II}\|_{L^{p}} \lesssim\left\|\frac{\omega_{\theta}}{r}\right\|_{L^{3,1} \cap L^{p}}\left(\sum_{i=1}^{2}\left\|x_{i} \rho\right\|_{B_{\infty, 1}^{0}}+\|\rho\|_{B_{p, 1}^{0} \cap L^{\infty}}\right) .
$$

Estimate of III. We now consider the remainder term. We separate it into 
two terms, the high frequency term and the low frequency term:

$$
\begin{aligned}
\mathrm{III} & =\sum_{j}\left[\Delta_{j}, R(v, \nabla)\right] \rho=\sum_{q \geq j-4}\left[\Delta_{j}, \Delta_{q} v \widetilde{\Delta}_{q} \nabla\right] \rho \\
& =\sum_{j=-1}^{3}\left[\Delta_{j}, \Delta_{-1} v \widetilde{\Delta}_{-1} \nabla\right] \rho+\sum_{\substack{q \geq j-4 \\
q \in \mathbb{N}}}\left[\Delta_{j}, \Delta_{q} v \widetilde{\Delta}_{q} \nabla\right] \rho=\mathrm{III}_{1}+\mathrm{III}_{2} .
\end{aligned}
$$

To treat the first term we use Proposition 2.7-b) and the Bernstein inequality:

$$
\begin{aligned}
\left\|\mathrm{III}_{1}\right\|_{L^{p}} & \leq \sum_{j=-1}^{3}\|x h j\|_{L^{1}}\left\|\nabla \Delta_{-1} v\right\|_{L^{\infty}}\left\|\widetilde{\Delta}_{-1} \nabla \rho\right\|_{L^{p}} \\
& \lesssim \sum_{j=-1}^{3} 2^{-j}\|x h\|_{L^{1}}\left\|\Delta_{-1} v\right\|_{L^{2}}\left\|\widetilde{\Delta}_{-1} \rho\right\|_{L^{p}} \lesssim\|v\|_{L^{2}}\|\rho\|_{L^{p}} .
\end{aligned}
$$

For the second term $\mathrm{III}_{2}$, we first write the term inside the sum as follows:

$$
\begin{aligned}
{\left[\Delta_{j}, \Delta_{q} v^{i} \widetilde{\Delta}_{q} \partial_{i}\right] \rho=} & \Delta_{j}\left(\Delta_{q} v^{i} \widetilde{\Delta}_{q} \partial_{i} \rho\right)-\Delta_{q} v^{i} \widetilde{\Delta}_{q} \partial_{i} \Delta_{j} \rho \\
= & \Delta_{j} \partial_{i}\left(\Delta_{q} v^{i} \widetilde{\Delta}_{q} \rho\right)-\Delta_{j}\left(\Delta_{q} \partial_{i} v^{i} \widetilde{\Delta}_{q} \rho\right) \\
& -\partial_{i}\left(\Delta_{q} v^{i} \widetilde{\Delta}_{q} \Delta_{j} \rho\right)+\Delta_{q} \partial_{i} v^{i} \widetilde{\Delta}_{q} \Delta_{j} \rho
\end{aligned}
$$

Summing over $i=\{1,2,3\}$ and using the incompressibility of the velocity, we get

$$
\sum_{i=1}^{3}\left[\Delta_{j}, \Delta_{q} v^{i} \widetilde{\Delta}_{q} \partial_{i}\right] \rho=\sum_{i=1}^{3}\left(\Delta_{j} \partial_{i}\left(\Delta_{q} v^{i} \widetilde{\Delta}_{q} \rho\right)-\partial_{i}\left(\Delta_{q} v^{i} \widetilde{\Delta}_{q} \Delta_{j} \rho\right)\right)
$$

Since

$$
\widetilde{\Delta}_{q} \Delta_{j} \rho=0 \text { if }|q-j| \geq 4 \text { and } \Delta_{j}\left(\Delta_{q} v^{i} \widetilde{\Delta}_{q} \rho\right)=0 \text { if } j \geq q+4
$$

we obtain

$$
\mathrm{III}_{2}=\sum_{i=1}^{3}\left(\sum_{\substack{q \geq j-4 \\ q \in \mathbb{N}}} \Delta_{j} \partial_{i}\left(\Delta_{q} v^{i} \widetilde{\Delta}_{q} \rho\right)-\sum_{\substack{|q-j| \leq 3 \\ q \in \mathbb{N}}} \partial_{i}\left(\Delta_{q} v^{i} \widetilde{\Delta}_{q} \Delta_{j} \rho\right)\right)=\sum_{i=1}^{3} \mathrm{III}_{2}^{i}
$$

We now estimate $\mathrm{III}_{2}^{1}$. First, decomposing as in (3.2),

$$
\begin{aligned}
\mathrm{III}_{2}^{1} & =\sum_{\substack{q \geq j-4, q \in \mathbb{N}}} \Delta_{j} \partial_{1}\left(\Delta_{q} v^{1} \widetilde{\Delta}_{q} \rho\right)-\sum_{\substack{|q-j| \leq 3, q \in \mathbb{N}}} \partial_{1}\left(\Delta_{q} v^{1} \widetilde{\Delta}_{q} \Delta_{j} \rho\right) \\
& =\mathrm{III}_{21}^{1}+\mathrm{III}_{22}^{1}+\mathrm{III}_{23}^{1}+\mathrm{III}_{24}^{1},
\end{aligned}
$$


where

$$
\begin{aligned}
& \mathrm{III}_{21}^{1}=\sum_{\substack{q \geq j-4, q \in \mathbb{N}}} \Delta_{j} \partial_{1}\left(\Delta_{q}\left(x_{1} \Delta^{-1} \partial_{3}\left(\frac{\omega_{\theta}}{r}\right)\right) \widetilde{\Delta}_{q} \rho\right) \\
& \mathrm{III}_{22}^{1}=-2 \sum_{\substack{q \geq j-4, q \in \mathbb{N}}} \Delta_{j} \partial_{1}\left(\Delta_{q} \partial_{13} \Delta^{-2}\left(\frac{\omega_{\theta}}{r}\right) \widetilde{\Delta}_{q} \rho\right) \\
& \mathrm{III}_{23}^{1}=-\sum_{\substack{|q-j| \leq 3, q \in \mathbb{N}}} \partial_{1}\left(\Delta_{q}\left(x_{1} \Delta^{-1} \partial_{3}\left(\frac{\omega_{\theta}}{r}\right)\right) \widetilde{\Delta}_{q} \Delta_{j} \rho\right) \\
& \mathrm{III}_{24}^{1}=2 \sum_{\substack{|q-j| \leq 3, q \in \mathbb{N}}} \partial_{1}\left(\Delta_{q} \partial_{13} \Delta^{-2}\left(\frac{\omega_{\theta}}{r}\right) \widetilde{\Delta}_{q} \Delta_{j} \rho\right) .
\end{aligned}
$$

Estimate of $\mathrm{III}_{21}^{1}$. To estimate the first term $\mathrm{III}_{21}^{1}$, we write, using (3.3),

$$
\begin{aligned}
\mathrm{III}_{21}^{1}= & \sum_{\substack{q \geq j-4, q \in \mathbb{N}}} \partial_{1} \Delta_{j}\left(\Delta_{q}\left(\Delta^{-1} \partial_{3}\left(\frac{\omega_{\theta}}{r}\right)\right) x_{1} \widetilde{\Delta}_{q} \rho\right) \\
& -\sum_{\substack{q \geq j-4, q \in \mathbb{N}}} \partial_{1} \Delta_{j}\left(\left(2^{2 q} \varphi_{1}\left(2^{q} \cdot\right) * \Delta^{-1} \partial_{3}\left(\frac{\omega_{\theta}}{r}\right)\right) \widetilde{\Delta}_{q} \rho\right) \\
:= & \mathrm{III}_{211}^{1}+\mathrm{III}_{212}^{1}+\mathrm{III}_{213}^{1},
\end{aligned}
$$

where

$$
\begin{aligned}
& \mathrm{III}_{211}^{1}=\sum_{\substack{q \geq j-4 \\
q \in \mathbb{N}}} \partial_{1} \Delta_{j}\left(\Delta_{q} \Delta^{-1} \partial_{3}\left(\frac{\omega_{\theta}}{r}\right) \widetilde{\Delta}_{q}\left(x_{1} \rho\right)\right) \\
& \mathrm{III}_{212}^{1}=\sum_{\substack{q \geq j-4 \\
q \in \mathbb{N}}} \partial_{1} \Delta_{j}\left(\Delta_{q} \Delta^{-1} \partial_{3}\left(\frac{\omega_{\theta}}{r}\right)\left(2^{2 q} \varphi_{1}\left(2^{q} \cdot\right) * \rho\right)\right) \\
& \mathrm{III}_{213}^{1}=-\sum_{\substack{q \geq j-4 \\
q \in \mathbb{N}}} \partial_{1} \Delta_{j}\left(\left(2^{2 q} \varphi_{1}\left(2^{q} \cdot\right) * \Delta^{-1} \partial_{3}\left(\frac{\omega_{\theta}}{r}\right)\right) \widetilde{\Delta}_{q} \rho\right) .
\end{aligned}
$$

To estimate the term $\mathrm{III}_{211}^{1}$, we use the Bernstein and Hölder inequalities and (3.8). We find

$$
\begin{aligned}
\left\|\mathrm{III}_{211}^{1}\right\|_{L^{p}} & \lesssim \sum_{\substack{q \geq j-4, q \in \mathbb{N}}} 2^{j}\left\|\Delta_{q} \Delta^{-1} \partial_{3}\left(\frac{\omega_{\theta}}{r}\right)\right\|_{L^{p}}\left\|\widetilde{\Delta}_{q}\left(x_{1} \rho\right)\right\|_{L^{\infty}} \\
& \lesssim \sum_{\substack{q \geq j-4, q \in \mathbb{N}}} 2^{j-q}\left\|\nabla \Delta_{q} \Delta^{-1} \partial_{3}\left(\frac{\omega_{\theta}}{r}\right)\right\|_{L^{p}}\left\|\widetilde{\Delta}_{q}\left(x_{1} \rho\right)\right\|_{L^{\infty}} \\
& \lesssim\left\|\frac{\omega_{\theta}}{r}\right\|_{L^{p}} \sum_{\substack{q \geq j-4 \\
q \in \mathbb{N}}} 2^{j-q}\left\|\widetilde{\Delta}_{q}\left(x_{1} \rho\right)\right\|_{L^{\infty}} \lesssim\left\|\frac{\omega_{\theta}}{r}\right\|_{L^{p}}\left\|x_{1} \rho\right\|_{B_{\infty, 1}^{0}} .
\end{aligned}
$$


Now to estimate the terms $\mathrm{III}_{212}^{1}$, we use the Young inequality and (3.8). We find

$$
\begin{aligned}
\left\|\operatorname{III}_{212}^{1}\right\|_{L^{p}} & \lesssim \sum_{\substack{q \geq j-4, q \in \mathbb{N}}} 2^{j}\left\|\Delta_{q} \Delta^{-1} \partial_{3}\left(\frac{\omega_{\theta}}{r}\right)\right\|_{L^{p}}\left\|2^{2 q} \varphi_{1}\left(2^{q} .\right) * \rho\right\|_{L^{\infty}} \\
& \lesssim\left\|\frac{\omega_{\theta}}{r}\right\|_{L^{p}} \sum_{\substack{q \geq j-4, q \in \mathbb{N}}} 2^{j-q} 2^{-q}\left\|\varphi_{1}\right\|_{L^{1}}\|\rho\|_{L^{\infty}} \lesssim\left\|\frac{\omega_{\theta}}{r}\right\|_{L^{p}}\|\rho\|_{L^{\infty}} .
\end{aligned}
$$

The term $\mathrm{III}_{213}^{1}$ can be estimated by using the Bernstein and Hölder inequalities, combined with the convolution inequality and (2.4):

$$
\begin{aligned}
\left\|\mathrm{III}_{213}^{1}\right\|_{L^{p}} & \lesssim \sum_{\substack{q \geq j-4, q \in \mathbb{N}}} 2^{j}\left\|2^{2 q} \varphi_{1}\left(2^{q} \cdot\right) * \Delta^{-1} \partial_{3}\left(\frac{\omega_{\theta}}{r}\right)\right\|_{L^{\infty}}\left\|\widetilde{\Delta}_{q} \rho\right\|_{L^{p}} \\
& \lesssim \sum_{\substack{q \geq j-4, q \in \mathbb{N}}} 2^{j-q}\left\|\varphi_{1}\right\|_{L^{1}}\left\|\Delta^{-1} \partial_{3}\left(\frac{\omega_{\theta}}{r}\right)\right\|_{L^{\infty}}\left\|\widetilde{\Delta}_{q} \rho\right\|_{L^{p}} \\
& \lesssim\left\|\frac{\omega_{\theta}}{r}\right\|_{L^{3,1}} \sum_{\substack{q \geq j-4, q \in \mathbb{N}}} 2^{j-q}\left\|\widetilde{\Delta}_{q} \rho\right\|_{L^{p}} \lesssim\left\|\frac{\omega_{\theta}}{r}\right\|_{L^{3,1}}\|\rho\|_{B_{p, 1}^{0}} .
\end{aligned}
$$

Thus we obtain

$$
\left\|\mathrm{III}_{21}^{1}\right\|_{L^{p}} \lesssim\left\|\frac{\omega_{\theta}}{r}\right\|_{L^{3,1} \cap L^{p}}\left(\left\|x_{1} \rho\right\|_{B_{\infty, 1}^{0}}+\|\rho\|_{B_{p, 1}^{0} \cap L^{\infty}}\right) .
$$

Estimate of $\mathrm{III}_{22}^{1}$. Thanks to the Bernstein inequality, we have that, for every $p \in[1, \infty]$ that,

$$
\left\|\Delta_{q} \partial_{13} \Delta^{-2} f\right\|_{L^{p}} \lesssim 2^{-2 q}\left\|\nabla^{2} \Delta_{q} \partial_{13} \Delta^{-2} f\right\|_{L^{p}} \lesssim 2^{-2 q}\|f\|_{L^{p}}, \quad \forall q \geq 0 .
$$

This yields

$$
\begin{aligned}
\left\|\mathrm{III}_{22}^{1}\right\|_{L^{p}} & \lesssim \sum_{\substack{q \geq j-4 \\
q \in \mathbb{N}}} 2^{j}\left\|\Delta_{q} \partial_{13} \Delta^{-2}\left(\frac{\omega_{\theta}}{r}\right)\right\|_{L^{p}}\left\|\widetilde{\Delta}_{q} \rho\right\|_{L^{\infty}} \\
& \lesssim\left\|\frac{\omega_{\theta}}{r}\right\|_{L^{p}} \sum_{\substack{q \geq j-4 \\
q \in \mathbb{N}}} 2^{j-q} 2^{-q}\left\|\widetilde{\Delta}_{q} \rho\right\|_{L^{\infty}} \lesssim\left\|\frac{\omega_{\theta}}{r}\right\|_{L^{p}}\|\rho\|_{L^{\infty}} .
\end{aligned}
$$

Estimate of $\mathrm{III}_{23}^{1}$. This term can be written in a manner similar to the term $\mathrm{III}_{21}^{1}$, and we obtain finally

$$
\mathrm{III}_{23}^{1}=\mathrm{III}_{231}^{1}+\mathrm{III}_{232}^{1}+\mathrm{III}_{233}^{1}+\mathrm{III}_{234}^{1},
$$


where

$$
\begin{aligned}
& \mathrm{III}_{231}^{1}=-\sum_{\substack{|q-j| \leq 3 \\
q \in \mathbb{N} \\
q \in}} \partial_{1}\left(\Delta_{q} \Delta^{-1} \partial_{3}\left(\frac{\omega_{\theta}}{r}\right) \widetilde{\Delta}_{q} \Delta_{j}\left(x_{1} \rho\right)\right) \\
& \mathrm{III}_{232}^{1}=-\sum_{\substack{|q-j| \leq 3 \\
q \in \mathbb{N}}} \partial_{1}\left(\Delta_{q} \Delta^{-1} \partial_{3}\left(\frac{\omega_{\theta}}{r}\right) \widetilde{\Delta}_{q}\left(2^{2 j} \varphi_{1}\left(2^{j} \cdot\right) * \rho\right)\right) \\
& \mathrm{III}_{233}^{1}=-\sum_{\substack{|q-j| \leq 3 \\
q \in \mathbb{N}}} \partial_{1}\left(\Delta_{q} \Delta^{-1} \partial_{3}\left(\frac{\omega_{\theta}}{r}\right)\left(2^{2 q} \varphi_{1}\left(2^{q} \cdot\right) * \Delta_{j} \rho\right)\right) \\
& \mathrm{III}_{234}^{1}=\sum_{\substack{|q-j| \leq 3, q \in \mathbb{N}}} \partial_{1}\left(\left(2^{2 q} \varphi_{1}\left(2^{q} \cdot\right) * \Delta^{-1} \partial_{3}\left(\frac{\omega_{\theta}}{r}\right)\right) \widetilde{\Delta}_{q} \Delta_{j} \rho\right) .
\end{aligned}
$$

We point out that, by reproducing the same analysis as for $\mathrm{III}_{21}^{1}$, we get

$$
\left\|\mathrm{III}_{23}^{1}\right\|_{L^{p}} \lesssim\left\|\frac{\omega_{\theta}}{r}\right\|_{L^{3,1} \cap L^{p}}\left(\left\|x_{1} \rho\right\|_{B_{\infty, 1}^{0}}+\|\rho\|_{B_{p, 1}^{0} \cap L^{\infty}}\right) .
$$

Estimate of $\mathrm{III}_{24}^{1}$. Using (3.12), we find

$$
\begin{aligned}
\left\|\mathrm{III}_{24}^{1}\right\|_{L^{p}} & \lesssim \sum_{\substack{|q-j| \leq 3 \\
q \in \mathbb{N}}} 2^{j}\left\|\Delta_{q} \partial_{13} \Delta^{-2}\left(\frac{\omega_{\theta}}{r}\right)\right\|_{L^{p}}\left\|\widetilde{\Delta}_{q} \Delta_{j} \rho\right\|_{L^{\infty}} \\
& \lesssim\left\|\frac{\omega_{\theta}}{r}\right\|_{L^{p}} \sum_{\substack{|q-j| \leq 3, q \in \mathbb{N}}} 2^{j-q} 2^{-q}\left\|\widetilde{\Delta}_{q} \rho\right\|_{L^{\infty}} \lesssim\left\|\frac{\omega_{\theta}}{r}\right\|_{L^{p}}\|\rho\|_{L^{\infty}} .
\end{aligned}
$$

Combining (3.11), (3.13), (3.14), and (3.15), we find finally

$$
\left\|\mathrm{III}_{2}^{1}\right\|_{L^{p}} \lesssim\left\|\frac{\omega_{\theta}}{r}\right\|_{L^{3,1} \cap L^{p}}\left(\left\|x_{1} \rho\right\|_{B_{\infty, 1}^{0}}+\|\rho\|_{B_{p, 1}^{0} \cap L^{\infty}}\right) .
$$

The term $\mathrm{III}_{2}^{2}$ can be estimated as was $\mathrm{III}_{2}^{1}$. For the term $\mathrm{III}_{2}^{3}$, we use (3.7) and then, reproducing the prior analysis, we get, with $x_{h}:=\left(x_{1}, x_{2}\right)$, the estimate

$$
\left\|\mathrm{III}_{2}^{2}\right\|_{L^{p}}+\left\|\mathrm{III}_{2}^{3}\right\|_{L^{p}} \lesssim\left\|\frac{\omega_{\theta}}{r}\right\|_{L^{3,1} \cap L^{p}}\left(\left\|x_{h} \rho\right\|_{B_{\infty, 1}^{0}}+\|\rho\|_{B_{p, 1}^{0} \cap L^{\infty}}\right) .
$$

Combining the above estimate with (3.16) yields

$$
\left\|\mathrm{III}_{2}\right\|_{L^{p}} \lesssim\left\|\frac{\omega_{\theta}}{r}\right\|_{L^{3,1} \cap L^{p}}\left(\left\|x_{h} \rho\right\|_{B_{\infty, 1}^{0}}+\|\rho\|_{B_{p, 1}^{0} \cap L^{\infty}}\right) .
$$

Now, from (3.10) and (3.17) we get

$$
\|\mathrm{III}\|_{L^{p}} \lesssim\|v\|_{L^{2}}\|\rho\|_{L^{p}}+\left\|\frac{\omega_{\theta}}{r}\right\|_{L^{3,1} \cap L^{p}}\left(\left\|x_{h} \rho\right\|_{B_{\infty, 1}^{0}}+\|\rho\|_{B_{p, 1}^{0} \cap L^{\infty}}\right) .
$$

This finishes the proof of the proposition. 


\section{Proof of Theorem 1.2}

To prove Theorem 1.2, we will restrict ourselves to proving some a priori estimates and the inviscid limit. The proof of the uniqueness and the existence of the solutions are standard.

\subsection{A priori estimates}

We establish in this subsection some global a priori estimates which we need in the proof of our main result. First we give some energy estimates and we shall prove the estimate of $\left\|v^{r} / r\right\|_{L^{\infty}}$, which is based on the estimation of our commutator in the previous section. Finally we will establish control of the Lipschitz norm of the velocity. We start with the energy estimates.

4.1.1. Energy estimates. We have the following estimates.

Proposition 4.1. Let $(v, \rho)$ be a smooth solution of (1.1). Then we have:

(a) For $\left(v^{0}, \rho^{0}\right) \in L^{2} \times L^{2}, t \in \mathbb{R}_{+}$, and $\nu \geq 0$,

$$
\|v(t)\|_{L^{2}}^{2}+2 \nu \int_{0}^{t}\|\nabla v(\tau)\|_{L^{2}}^{2} d \tau \leq C_{0}\left(1+t^{2}\right),
$$

where $C_{0}$ depends on $\left\|v^{0}\right\|_{L^{2}}$ and $\left\|\rho^{0}\right\|_{L^{2}}$ but not on the viscosity $\nu$.

(b) For $\rho^{0} \in L^{2}$,

$$
\|\rho\|_{L_{t}^{\infty} L^{2}}^{2}+2\|\nabla \rho\|_{L_{t}^{2} L^{2}}^{2}=\left\|\rho^{0}\right\|_{L^{2}}^{2} \quad \text { and } \quad\|\rho(t)\|_{L^{\infty}} \leq C t^{-3 / 4}\left\|\rho^{0}\right\|_{L^{2}} .
$$

The constant $C$ does not depend on the viscosity.

Note that the axisymmetry of the velocity and the density are not needed in this proposition. The proof of the first estimate (a) can be found in [3]. For the proof of (b), see [12].

We aim now to give some estimates of the horizontal moment $x_{h} \rho$ of the density that will be needed later. See Proposition 4.2 (1)-(3) in [12] for a proof.

Proposition 4.2. Let $v$ be a smooth vector field with zero divergence and let $\rho$ be a smooth solution of the second equation of (1.1). Then we have:

(1) If $\rho^{0} \in L^{2}$ and $x_{h} \rho^{0} \in L^{2}$, there exists $C_{0}>0$ such that, for every $t \in \mathbb{R}_{+}$,

$$
\left\|x_{h} \rho\right\|_{L_{t}^{\infty} L^{2}}+\left\|x_{h} \rho\right\|_{L_{t}^{2} \dot{H}^{1}} \leq C_{0}\left(1+t^{5 / 4}\right) .
$$

(2) If $\rho^{0} \in L^{2}$ and $\left|x_{h}\right|^{2} \rho^{0} \in L^{2}$, there exists $C_{0}>0$ such that, for every $t \in \mathbb{R}_{+}$,

$$
\left\|\left|x_{h}\right|^{2} \rho\right\|_{L_{t}^{\infty} L^{2}}+\left\|\left|x_{h}\right|^{2} \rho\right\|_{L_{t}^{2} \dot{H}^{1}} \leq C_{0}\left(1+t^{5 / 2}\right),
$$

where $C_{0}$ depend only on the norm of the initial data and not on the viscosity. 
4.1.2. Strong estimates. We will prove in the first step a bound for $\left\|\frac{\omega}{r}(t)\right\|_{L^{3,1}}$ which is the quantity important for obtaining the global existence of smooth solutions. It allows us to bound the vorticity in $L^{\infty}$ space for all times and then to bound the Lipschitz norm of the velocity $\|\nabla v(t)\|_{L^{\infty}}$.

Proposition 4.3. Let $v^{0}$ be a smooth axisymmetric vector field with zero divergence such that $v^{0} \in L^{2}$, let its vorticity be such that $\omega^{0} / r \in L^{2} \cap L^{\bar{p}}$ with $3<\bar{p}<6$, and let $\rho^{0} \in B_{2,1}^{0} \cap B_{\bar{p}, 1}^{0} \cap L^{m}$, with $m>6$, be an axisymmetric function such that $\left|x_{h}\right|^{2} \rho^{0} \in L^{2}$. Then for every $t \in \mathbb{R}_{+}$, we have

$$
\left\|\frac{\omega}{r}(t)\right\|_{L^{3,1}}+\left\|\frac{v^{r}}{r}(t)\right\|_{L^{\infty}} \leq \Phi_{2}(t)
$$

We recall that $\Phi_{2}(t)=C_{0} e^{\exp \left\{C_{0} t^{19 / 6}\right\}}$ and the constant $C_{0}$ depends only on the norm of the initial data but not on the viscosity $\nu$.

Remark 4.4. We note that for $\rho^{0} \in H^{s-2}$ with $s>5 / 2$, there exists $\bar{p}>3$ such that $\rho^{0} \in B_{2,1}^{0} \cap B_{\bar{p}, 1}^{0}$.

Proof. We start with the following result proved in [2]:

$$
\left|v^{r} / r\right| \lesssim \frac{1}{|\cdot|^{2}} *\left|\frac{\omega_{\theta}}{r}\right|
$$

Using Lemma 2.9 and (2.3), we have, for $\bar{p}>3$,

$$
\left\|v^{r} / r\right\|_{L^{\infty}} \lesssim\left\|\frac{1}{|\cdot|^{2}}\right\|_{L^{3 / 2, \infty}}\left\|\frac{\omega_{\theta}}{r}\right\|_{L^{3,1}} \lesssim\left\|\frac{\omega_{\theta}}{r}\right\|_{L^{3,1}} \lesssim\left\|\frac{\omega_{\theta}}{r}\right\|_{L^{2} \cap L^{\bar{p}}} .
$$

There remains to estimate $\left\|\omega_{\theta} / r\right\|_{L^{2} \cap L^{\bar{p}}}$. For this purpose we recall that the function $\zeta:=\omega_{\theta} / r$ satisfies the equation

$$
\partial_{t} \zeta+v \cdot \nabla \zeta-\nu\left(\Delta+\frac{2}{r} \partial_{r}\right) \zeta=-\frac{\partial_{r} \rho}{r} .
$$

By making $L^{p}$ estimates, we get

$$
\|\zeta(t)\|_{L^{2} \cap L^{\bar{p}}} \leq\left\|\zeta^{0}\right\|_{L^{2} \cap L^{\bar{p}}}+\int_{0}^{t}\left\|\frac{\partial_{r} \rho}{r}(\tau)\right\|_{L^{2} \cap L^{\bar{p}}} d \tau .
$$

At this stage we need the following lemma. We refer to [12] for the proof.

Lemma 4.5. For every axisymmetric smooth scalar function u, we have

$$
\frac{\partial_{r}}{r} u=\sum_{i, j=1}^{2} b_{i j}(x) \partial_{i j} u
$$

where the functions $b_{i j}$ are bounded.

Consequently, for every $1 \leq p \leq \infty$, we obtain

$$
\left\|\frac{\partial_{r}}{r} u\right\|_{L^{p}} \lesssim\left\|\nabla^{2} u\right\|_{L^{p}}
$$


Using Lemma 4.5, the Bernstein inequality, and Lemma 2.10 we obtain

$$
\begin{aligned}
\left\|\frac{\partial_{r} \rho}{r}\right\|_{L_{t}^{1}\left(L^{2} \cap L^{\bar{p}}\right)} \lesssim\left\|\nabla^{2} \rho\right\|_{L_{t}^{1}\left(L^{2} \cap L^{\bar{p}}\right)} \lesssim \sum_{j \geq-1}\left\|\Delta_{j} \nabla^{2} \rho\right\|_{L_{t}^{1}\left(L^{2} \cap L^{\bar{p}}\right)} \\
\quad \lesssim \sum_{j \geq-1} 2^{2 j}\left\|\Delta_{j} \rho\right\|_{L_{t}^{1}\left(L^{2} \cap L^{\bar{p}}\right)} \\
\quad \lesssim \int_{0}^{t}\left\|\Delta_{-1} \rho(\tau)\right\|_{L^{2} \cap L^{\bar{p}}} d \tau+\sum_{j \geq 0} 2^{2 j} \int_{0}^{t}\left\|\Delta_{j} \rho(\tau)\right\|_{L^{2} \cap L^{\bar{p}}} d \tau \\
\quad \lesssim t\left\|\rho^{0}\right\|_{L^{2} \cap L^{\bar{p}}}+\sum_{j \geq 0} 2^{2 j} \int_{0}^{t}\left\|\Delta_{j} \rho(\tau)\right\|_{L^{2} \cap L^{\bar{p}}} d \tau .
\end{aligned}
$$

Now use Proposition 2.12 to obtain

$$
\begin{aligned}
\left\|\frac{\partial_{r} \rho}{r}\right\|_{L_{t}^{1}\left(L^{2} \cap L^{\bar{p}}\right)} & \lesssim t\left\|\rho^{0}\right\|_{L^{2} \cap L^{\bar{p}}}+\sum_{j \geq 0}\left(\left\|\Delta_{j} \rho^{0}\right\|_{L^{2} \cap L^{\bar{p}}}+\left\|\left[\Delta_{j}, v \cdot \nabla\right] \rho\right\|_{L_{t}^{1}\left(L^{2} \cap L^{\bar{p}}\right)}\right) \\
& \lesssim\left\|\rho^{0}\right\|_{B_{2,1}^{0} \cap B_{\bar{p}, 1}^{0}}(1+t)+\sum_{j \geq 0}\left\|\left[\Delta_{j}, v \cdot \nabla\right] \rho\right\|_{L_{t}^{1}\left(L^{2} \cap L^{\bar{p}}\right)^{.}} .
\end{aligned}
$$

Therefore, using Proposition 3.2, we obtain

$$
\begin{aligned}
\left\|\frac{\partial_{r} \rho}{r}\right\|_{L_{t}^{1}\left(L^{2} \cap L^{\bar{p}}\right)} \lesssim & \left\|\rho^{0}\right\|_{B_{2,1}^{0} \cap B_{\bar{p}, 1}^{0}}(1+t)+\int_{0}^{t}\|v(\tau)\|_{L^{2}}\|\rho(\tau)\|_{L^{2} \cap L^{\bar{p}}} d \tau \\
& +\int_{0}^{t}\|\zeta(\tau)\|_{L^{2} \cap L^{\bar{p}}}\left(\left\|x_{h} \rho(\tau)\right\|_{B_{\infty, 1}^{0}}+\|\rho(\tau)\|_{B_{2,1}^{0} \cap B_{\bar{p}, 1}^{0} \cap L^{\infty}}\right) d \tau \\
\lesssim & \left\|\rho^{0}\right\|_{B_{2,1}^{0} \cap B_{\bar{p}, 1}^{0}}(1+t)+\left\|\rho^{0}\right\|_{L^{2} \cap L^{\bar{p}}} \int_{0}^{t}\|v(\tau)\|_{L^{2}} d \tau \\
& +\int_{0}^{t}\|\zeta(\tau)\|_{L^{2} \cap L^{\bar{p}}}\left(\left\|x_{h} \rho(\tau)\right\|_{B_{\infty, 1}^{0}}+\|\rho(\tau)\|_{B_{2,1}^{0} \cap B_{\bar{p}, 1}^{0} \cap L^{\infty}}\right) d \tau .
\end{aligned}
$$

Substituting this last estimate into (4.2) and using Proposition 4.1, we get

$$
\begin{aligned}
\|\zeta(t)\|_{L^{2} \cap L^{\bar{p}}} \lesssim & \left\|\zeta^{0}\right\|_{L^{2} \cap L^{\bar{p}}}+\left\|\rho^{0}\right\|_{B_{2,1}^{0} \cap B_{\bar{p}, 1}^{0}}(1+t)+\left\|\rho^{0}\right\|_{L^{2} \cap L^{\bar{p}}} C_{0} t(1+t) \\
& +\int_{0}^{t}\|\zeta(\tau)\|_{L^{2} \cap L^{\bar{p}}}\left(\left\|x_{h} \rho(\tau)\right\|_{B_{\infty, 1}^{0}}+\|\rho(\tau)\|_{B_{2,1}^{0} \cap B_{\bar{p}, 1}^{0} \cap L^{\infty}}\right) d \tau .
\end{aligned}
$$

Gronwall's inequality gives

$$
\|\zeta(t)\|_{L^{2} \cap L^{\bar{p}}} \leq C_{0}\left(1+t^{2}\right) \exp \left\{C\left\|x_{h} \rho\right\|_{L_{t}^{1} B_{\infty, 1}^{0}}+C\|\rho\|_{L_{t}^{1}\left(B_{2,1}^{0} \cap B_{\bar{p}, 1}^{0} \cap L^{\infty}\right)}\right\} .
$$

To estimate the term $\|\rho\|_{L_{t}^{1} B_{2,1}^{0}}$, we use the embedding $B_{2,1}^{1 / 2} \hookrightarrow B_{2,1}^{0}$, the interpolation estimate, the Hölder inequality and Proposition 4.1:

$$
\|\rho\|_{L_{t}^{1} B_{2,1}^{0}} \lesssim\|\rho\|_{L_{t}^{1} B_{2,1}^{1 / 2}} \lesssim\|\rho\|_{L_{t}^{\infty} L^{2}}^{1 / 2} t^{3 / 4}\|\nabla \rho\|_{L_{t}^{2} L^{2}}^{1 / 2} \lesssim t^{3 / 4}\left\|\rho^{0}\right\|_{L^{2}} .
$$


The term $\|\rho\|_{L_{t}^{1} L^{\infty}}$, can be estimated using the second estimate of Proposition 4.1-(b) and integrating in time. We get

$$
\|\rho\|_{L_{t}^{1} L^{\infty}} \lesssim t^{1 / 4}\left\|\rho^{0}\right\|_{L^{2}}
$$

and, for $\|\rho\|_{L_{t}^{1} B_{\bar{p}, 1}^{0}}$, we have by the definition of the Besov spaces and for $2<\bar{p}<6$ and Proposition 4.1-(b), that, for $2<\bar{p}<6$,

$$
\begin{aligned}
\|\rho\|_{L_{t}^{1} B_{\bar{p}, 1}^{0}} & =\sum_{q \geq-1}\left\|\Delta_{q} \rho\right\|_{L_{t}^{1} L^{\bar{p}}} \lesssim \sum_{q \geq-1} 2^{3 q(1 / 2-1 / \bar{p})}\left\|\Delta_{q} \rho\right\|_{L_{t}^{1} L^{2}} \\
& \lesssim \sum_{q \geq-1} 2^{q(1 / 2-3 / \bar{p})} 2^{q}\left\|\Delta_{q} \rho\right\|_{L_{t}^{1} L^{2}} \lesssim\|\rho\|_{L_{t}^{1} H^{1}} \lesssim t^{1 / 2}\|\rho\|_{L_{t}^{2} H^{1}} \lesssim t^{1 / 2}\left\|\rho^{0}\right\|_{L^{2}}
\end{aligned}
$$

Consequently, in light of (4.3), we obtain

$$
\begin{aligned}
\|\zeta(t)\|_{L^{2} \cap L^{\bar{p}}} & \leq C_{0}\left(1+t^{2}\right) e^{C\left(t^{3 / 4}+t^{1 / 2}+t^{1 / 4}\right)\left\|\rho^{0}\right\|_{L^{2}}} e^{C\left\|x_{h} \rho\right\|_{L_{t}^{1} B_{\infty, 1}^{0}}} \\
& \leq C_{0} e^{C_{0} t^{2}} e^{C\left\|x_{h} \rho\right\|_{L_{t}^{1} B_{\infty, 1}^{0}}} .
\end{aligned}
$$

To estimate the term $\left\|x_{h} \rho\right\|_{L_{t}^{1} B_{\infty, 1}^{0}}$, we use the following inequality proved for $\rho^{0} \in L^{2} \cap L^{m}$ with $m>6$ and $\left|x_{h}\right|^{2} \rho^{0} \in L^{2}$ (see [12] for a proof):

$$
\left\|x_{h} \rho\right\|_{L_{t}^{1} B_{\infty, 1}^{0}} \leq C_{0}\left(1+t^{19 / 6}\right)+C_{0} \int_{0}^{t}\left(\tau^{13 / 6}+\tau^{-3 / 4}\right) \log \left(2+\|\zeta\|_{L_{\tau}^{\infty} L^{3,1}}\right) d \tau .
$$

Hence, for $\bar{p}>3$, we get

(4.5) $\left\|x_{h} \rho\right\|_{L_{t}^{1} B_{\infty, 1}^{0}} \leq C_{0}\left(1+t^{19 / 6}\right)+C_{0} \int_{0}^{t}\left(\tau^{13 / 6}+\tau^{-3 / 4}\right) \log \left(2+\|\zeta\|_{L_{\tau}^{\infty}\left(L^{2} \cap L^{\bar{p}}\right.}\right) d \tau$.

Combining (4.4) and (4.5), we find that

$$
\begin{aligned}
& \log \left(2+\|\zeta\|_{L_{\tau}^{\infty}\left(L^{2} \cap L^{\bar{p}}\right)}\right) \\
& \quad \leq C_{0}\left(1+t^{19 / 6}\right)+C_{0} \int_{0}^{t}\left(\tau^{13 / 6}+\tau^{-3 / 4}\right) \log \left(2+\|\zeta\|_{L_{\tau}^{\infty}\left(L^{2} \cap L^{\bar{p}}\right)}\right) d \tau .
\end{aligned}
$$

Gronwall's inequality gives

$$
\log \left(2+\|\zeta\|_{L_{\tau}^{\infty}\left(L^{2} \cap L^{\bar{p}}\right)}\right) \leq C_{0}\left(1+t^{19 / 6}\right) e^{C_{0}\left(t^{19 / 6}+t^{1 / 4}\right)} \leq \Phi_{1}(t) .
$$

Therefore, using (4.5) once more, we get

$$
\left\|x_{h} \rho\right\|_{L_{t}^{1} B_{\infty, 1}^{0}} \leq \Phi_{1}(t) .
$$

This yields in (4.4) that

$$
\|\zeta(t)\|_{L^{2} \cap L^{\bar{p}}} \leq \Phi_{2}(t) .
$$

Hence, it follows from (4.1) that,

$$
\|\zeta(t)\|_{L^{3,1}} \leq \Phi_{2}(t) .
$$


Then, with $\omega=\omega_{\theta} e_{\theta}$, using (2.2), we have

$$
\left\|\frac{\omega}{r}(t)\right\|_{L^{3,1}} \leq\|\zeta(t)\|_{L^{3,1}} \leq \Phi_{2}(t)
$$

Thanks to (4.1), we obtain

$$
\left\|\frac{v^{r}}{r}(t)\right\|_{L^{\infty}} \leq \Phi_{2}(t)
$$

This finishes the proof of the proposition.

Now we will use the above estimates to obtain a bound for $\|\omega(t)\|_{L^{\infty}}$.

Proposition 4.6. Suppose the hypotheses of Proposition 4.3 and, additionally, that $\omega^{0} \in L^{\infty}$. Then, for every $t \in \mathbb{R}_{+}$, we have

$$
\|\omega(t)\|_{L^{\infty}}+\|\nabla \rho\|_{L_{t}^{1} L^{\infty}} \leq \Phi_{4}(t) .
$$

We recall that $\Phi_{4}(t)$ does not depend on the viscosity.

Proof. Recall that the vorticity $\omega$ satisfies the equation

$$
\partial_{t} \omega+v \cdot \nabla \omega-\nu \Delta \omega=\frac{v^{r}}{r} \omega+\operatorname{curl}\left(\rho e_{z}\right)
$$

Applying the maximum principle and using Proposition 4.3,

$$
\begin{aligned}
\|\omega(t)\|_{L^{\infty}} & \leq\left\|\omega^{0}\right\|_{L^{\infty}}+\int_{0}^{t}\left\|\frac{v^{r}}{r}(\tau)\right\|_{L^{\infty}}\|\omega(\tau)\|_{L^{\infty}} d \tau+\int_{0}^{t}\left\|\operatorname{curl}\left(\rho e_{z}\right)(\tau)\right\|_{L^{\infty}} d \tau \\
& \leq\left\|\omega^{0}\right\|_{L^{\infty}}+\int_{0}^{t} \Phi_{2}(\tau)\|\omega(\tau)\|_{L^{\infty}} d \tau+\int_{0}^{t}\|\nabla \rho(\tau)\|_{L^{\infty}} d \tau .
\end{aligned}
$$

By Gronwall's inequality this implies

$$
\|\omega(t)\|_{L^{\infty}} \leq\left(\left\|\omega^{0}\right\|_{L^{\infty}}+\int_{0}^{t}\|\nabla \rho(\tau)\|_{L^{\infty}} d \tau\right) \Phi_{3}(t) .
$$

There remains to estimate $\|\nabla \rho\|_{L_{t}^{1} L^{\infty}}$. To this end, using the Bernstein inequality for $\bar{p}>3$, we obtain

$$
\begin{aligned}
\|\nabla \rho\|_{L_{t}^{1} L^{\infty}} & \leq\left\|\nabla \Delta_{-1} \rho\right\|_{L_{t}^{1} L^{\infty}}+\sum_{j \geq 0}\left\|\nabla \Delta_{j} \rho\right\|_{L_{t}^{1} L^{\infty}} \\
& \lesssim\|\rho\|_{L_{t}^{1} L^{2}}+\sum_{j \geq 0} 2^{j(3 / \bar{p}+1)}\left\|\Delta_{j} \rho\right\|_{L_{t}^{1} L^{\bar{p}}}
\end{aligned}
$$


Using now Proposition 2.11, Proposition 4.1, and the Bernstein inequality for $\bar{p}>3$,

$$
\begin{aligned}
\|\nabla \rho\|_{L_{t}^{1} L^{\infty}} \lesssim & \left\|\rho^{0}\right\|_{L^{2}} t+\sum_{j \geq 0} 2^{j(3 / \bar{p}-1)}\left\|\rho^{0}\right\|_{L^{\bar{p}}}\left(1+(j+1) \int_{0}^{t}\|\omega(\tau)\|_{L^{\infty}} d \tau\right) \\
& +\sum_{j \geq 0} 2^{j(3 / \bar{p}-1)}\left\|\rho^{0}\right\|_{L^{\bar{p}}} \int_{0}^{t}\left\|\nabla \Delta_{-1} v(\tau)\right\|_{L^{\infty}} d \tau \\
\lesssim & \left\|\rho^{0}\right\|_{L^{2}} t+\left\|\rho^{0}\right\|_{L^{\bar{p}}}\left(1+\int_{0}^{t}\|\omega(\tau)\|_{L^{\infty}} d \tau+\int_{0}^{t}\|v(\tau)\|_{L^{2}} d \tau\right) \\
\lesssim & \left\|\rho^{0}\right\|_{L^{2}} t+\left\|\rho^{0}\right\|_{L^{\bar{p}}}\left(1+t\|v\|_{L_{t}^{\infty} L^{2}}+\int_{0}^{t}\|\omega(\tau)\|_{L^{\infty}} d \tau\right) \\
\lesssim & \left\|\rho^{0}\right\|_{B_{2,1}^{0} \cap B_{\bar{p}, 1}^{0}}\left(1+t+C_{0} t(1+t)+\int_{0}^{t}\|\omega(\tau)\|_{L^{\infty}} d \tau\right) \\
\leq & C_{0}\left(1+t^{2}+\int_{0}^{t}\|\omega(\tau)\|_{L^{\infty}} d \tau\right) .
\end{aligned}
$$

Substituting (4.7) into (4.6) and using Gronwall's inequality, we obtain

$$
\|\omega\|_{L^{\infty}} \lesssim\left(\left\|\omega^{0}\right\|_{L^{\infty}}+C_{0}\left(1+t^{2}+\int_{0}^{t}\|\omega(\tau)\|_{L^{\infty}} d \tau\right)\right) \Phi_{3}(t) \leq \Phi_{4}(t) .
$$

In (4.7) this gives

$$
\|\nabla \rho\|_{L_{t}^{1} L^{\infty}} \leq \Phi_{4}(t)
$$

which is the desired result.

Now we will propagate the subcritical Sobolev regularities globally in time. This is based on the estimate of $\|\nabla v(t)\|_{L^{\infty}}$. More precisely, we prove the following proposition.

Proposition 4.7. Let $(v, \rho)$ be a smooth solution of the stratified system (1.1) with $\nu \geq 0$, and such that $\left(v^{0}, \rho^{0}\right) \in H^{s} \times H^{s-2}$ with $s>5 / 2$. Then there exists $\Psi \in \mathcal{U}$ such that $\left(v^{0}, \rho^{0}\right) \in H^{s, \Psi} \times H^{s-2, \Psi}$ and, for every $t \in \mathbb{R}_{+}$,

$$
\begin{aligned}
\|v\|_{\widetilde{L}_{t}^{\infty} H^{s, \Psi}}+\|\rho\|_{\widetilde{L}_{t}^{\infty} H^{s-2, \Psi}}+\|\rho\|_{\widetilde{L}_{t}^{1} H^{s, \Psi}} \lesssim & \left(\left\|v^{0}\right\|_{H^{s, \Psi}}+\left\|\rho^{0}\right\|_{H^{s-2, \Psi}}(1+t)\right) \\
& \exp \left\{C\left(\|\nabla v\|_{L_{t}^{1} L^{\infty}}+\|\nabla \rho\|_{L_{t}^{1} L^{\infty}}\right)\right\} .
\end{aligned}
$$

If, in addition, $\rho^{0} \in L^{m}$ with $m>6$ and $\left|x_{h}\right|^{2} \rho^{0} \in L^{2}$, then for every $t \geq 0$, we get

$$
\|\nabla v(t)\|_{L^{\infty}} \leq \Phi_{5}(t), \quad\|v\|_{\widetilde{L}_{t}^{\infty} H^{s, \Psi}}+\|\rho\|_{\widetilde{L}_{t}^{\infty} H^{s-2, \Psi}}+\|\rho\|_{\widetilde{L}_{t}^{1} H^{s, \Psi}} \leq \Phi_{6}(t) .
$$

The constants $C, \Phi_{5}(t)$, and $\Phi_{6}(t)$ do not depend on the viscosity.

Remark 4.8. From Definition 2.5, we observe that when the profile $\Psi$ is a nonnegative constant, then $H^{s, \Psi}=H^{s}$. In this case, we get the global persistence of the Sobolev regularities

$$
\|\nabla v(t)\|_{L^{\infty}} \leq \Phi_{5}(t), \quad\|v\|_{\widetilde{L}_{t}^{\infty} H^{s}}+\|\rho\|_{\widetilde{L}_{t}^{\infty} H^{s-2}}+\|\rho\|_{\widetilde{L}_{t}^{1} H^{s}} \leq \Phi_{6}(t) .
$$

Recall that $\Phi_{5}(t)$ and $\Phi_{6}(t)$ do not depend on the viscosity. 
Proof. We localize in frequency the equation of the velocity. Then we have, for every $j \geq-1$,

$$
\partial_{t} \Delta_{j} v+v \cdot \nabla \Delta_{j} v-\nu \Delta \Delta_{j} v+\nabla \Delta_{j} p=\Delta_{j} \rho e_{z}-\left[\Delta_{j}, v \cdot \nabla\right] v .
$$

Taking the $L^{2}$ - scalar product of the above equation with $\Delta_{j} v$ and using the Hölder inequality,

$$
\frac{1}{2} \frac{d}{d t}\left\|\Delta_{j} v(t)\right\|_{L^{2}}^{2}+\nu\left\|\nabla \Delta_{j} v\right\|_{L^{2}}^{2} \leq\left\|\Delta_{j} v(t)\right\|_{L^{2}}\left(\left\|\Delta_{j} \rho(t)\right\|_{L^{2}}+\left\|\left[\Delta_{j}, v \cdot \nabla\right] v(t)\right\|_{L^{2}}\right) .
$$

Then,

$$
\frac{d}{d t}\left\|\Delta_{j} v(t)\right\|_{L^{2}} \leq\left\|\Delta_{j} \rho(t)\right\|_{L^{2}}+\left\|\left[\Delta_{j}, v \cdot \nabla\right] v(t)\right\|_{L^{2}}
$$

Integrating in time we obtain

$$
\left\|\Delta_{j} v(t)\right\|_{L^{2}} \leq\left\|\Delta_{j} v^{0}\right\|_{L^{2}}+\left\|\Delta_{j} \rho\right\|_{L_{t}^{1} L^{2}}+\left\|\left[\Delta_{j}, v \cdot \nabla\right] v\right\|_{L_{t}^{1} L^{2}} .
$$

Multiplying this inequality by $\Psi(j) 2^{s j}$ and taking the $\ell^{2}$-norm, we get

$$
\|v\|_{\widetilde{L}_{t}^{\infty} H^{s, \Psi}} \leq\left\|v^{0}\right\|_{H^{s, \Psi}}+\|\rho\|_{\widetilde{L}_{t}^{1} H^{s, \Psi}}+\left(\Psi(j) 2^{s j}\left\|\left[\Delta_{j}, v \cdot \nabla\right] v\right\|_{L_{t}^{1} L^{2}}\right)_{\ell^{2}} .
$$

Combining Lemma 2.4 with Proposition 3.1 we get,

$$
\begin{aligned}
\left(\Psi(j) 2^{s j}\left\|\left[\Delta_{j}, v \cdot \nabla\right] v\right\|_{L_{t}^{1} L^{2}}\right)_{\ell^{2}} & \lesssim \int_{0}^{t}\left(\Psi(j) 2^{s j}\left\|\left[\Delta_{j}, v \cdot \nabla\right] v(\tau)\right\|_{L^{2}}\right)_{\ell^{2}} d \tau \\
& \lesssim \int_{0}^{t}\|\nabla v(\tau)\|_{L^{\infty}}\|v(\tau)\|_{H^{s, \Psi}} d \tau
\end{aligned}
$$

Therefore, we get

$$
\|v\|_{\widetilde{L}_{t}^{\infty} H^{s, \Psi}} \leq\left\|v^{0}\right\|_{H^{s, \Psi}}+\|\rho\|_{\widetilde{L}_{t}^{1} H^{s, \Psi}}+C \int_{0}^{t}\|\nabla v(\tau)\|_{L^{\infty}}\|v(\tau)\|_{H^{s, \Psi}} d \tau .
$$

Now, to estimate $\|\rho\|_{\widetilde{L}_{t}^{1} H^{s, \Psi}}$, we use Proposition 2.12 for $j \geq 0$ :

$$
\left\|\Delta_{j} \rho\right\|_{L_{t}^{\infty} L^{2}}+2^{2 j}\left\|\Delta_{j} \rho\right\|_{L_{t}^{1} L^{2}} \lesssim\left\|\Delta_{j} \rho^{0}\right\|_{L^{2}}+\left\|\left[\Delta_{j}, v \cdot \nabla\right] \rho\right\|_{L_{t}^{1} L^{2}} .
$$

Multiplying this last inequality by $\Psi(j) 2^{j(s-2)}$, taking the $\ell^{2}$ norm, and using the Hölder inequality and Proposition 4.1-(b), we find

$$
\begin{aligned}
& \|\rho\|_{\widetilde{L}_{t}^{\infty} H^{s-2, \Psi}}+\|\rho\|_{\widetilde{L}_{t}^{1} H^{s, \Psi}} \\
& \quad \leq\left\|\Delta_{-1} \rho\right\|_{L_{t}^{\infty} L^{2}}+\left\|\Delta_{-1} \rho\right\|_{L_{t}^{1} L^{2}}+\left\|\rho^{0}\right\|_{H^{s-2, \Psi}}+\left\|\Psi(j) 2^{j(s-2)}\right\|\left[\Delta_{j}, v \cdot \nabla\right] \rho\left\|_{L_{t}^{1} L^{2}}\right\|_{\ell^{2}} \\
& \quad \leq C t\left\|\rho^{0}\right\|_{L^{2}}+C\left\|\rho^{0}\right\|_{H^{s-2, \Psi}}+\left\|\Psi(j) 2^{j(s-2)}\right\|\left[\Delta_{j}, v \cdot \nabla\right] \rho\left\|_{L_{t}^{1} L^{2}}\right\|_{\ell^{2}} .
\end{aligned}
$$

Since $0<s-2$, using Proposition 3.1, we obtain that

$$
\begin{aligned}
\left\|\left(\Psi(j) 2^{j(s-2)}\left\|\left[\Delta_{j}, v \cdot \nabla\right] \rho\right\|_{L_{t}^{1} L^{2}}\right)_{j}\right\|_{\ell^{2}} \leq & C \int_{0}^{t}\|\nabla v(\tau)\|_{L^{\infty}}\|\rho(\tau)\|_{H^{s-2, \Psi}} d \tau \\
& +C \int_{0}^{t}\|\nabla \rho(\tau)\|_{L^{\infty}}\|v(\tau)\|_{H^{s-2, \Psi}} d \tau .
\end{aligned}
$$


Therefore, using the embeddings $H^{s, \Psi} \hookrightarrow H^{s-2, \Psi}$, we find

$$
\begin{aligned}
\|\rho\|_{\widetilde{L}_{t}^{\infty} H^{s-2, \Psi}}+\|\rho\|_{\widetilde{L}_{t}^{1} H^{s, \Psi}} \lesssim & \left\|\rho^{0}\right\|_{L^{2}} t+\left\|\rho^{0}\right\|_{H^{s-2, \Psi}} \\
& +\int_{0}^{t}\|\nabla \rho(\tau)\|_{L^{\infty}}\|v(\tau)\|_{\widetilde{L}_{\tau}^{\infty} H^{s, \Psi}} d \tau \\
& +\int_{0}^{t}\|\nabla v(\tau)\|_{L^{\infty}}\|\rho(\tau)\|_{\widetilde{L}_{\tau}^{\infty} H^{s-2, \Psi}} d \tau .
\end{aligned}
$$

Setting $f(t):=\|v\|_{\widetilde{L}_{t}^{\infty} H^{s, \Psi}}+\|\rho\|_{\widetilde{L}_{t}^{\infty} H^{s-2, \Psi}}+\|\rho\|_{\widetilde{L}_{t}^{1} H^{s, \Psi}}$ and combining (4.8) and (4.9) with Gronwall's inequality, we obtain

$$
f(t) \lesssim\left(\left\|v^{0}\right\|_{H^{s, \Psi}}+\left\|\rho^{0}\right\|_{L^{2}} t+\left\|\rho^{0}\right\|_{H^{s-2, \Psi}}\right) e^{C\left(\|\nabla v\|_{L_{t}^{1} L^{\infty}}+\|\nabla \rho\|_{L_{t}^{1} L^{\infty}}\right)} .
$$

To estimate the term $\|\nabla \rho\|_{L_{t}^{1} L^{\infty}}$, we use Proposition 4.6 and to estimate the Lipschitz norm of the velocity we use the classical logarithmic estimate; for $s>5 / 2$,

$$
\begin{aligned}
\|\nabla v\|_{L^{\infty}} & \lesssim\|v\|_{L^{2}}+\|\omega\|_{L^{\infty}} \log \left(e+\|v\|_{H^{s}}\right) \\
& \lesssim\|v\|_{L^{2}}+\|\omega\|_{L^{\infty}} \log \left(e+\|v\|_{\widetilde{L}_{t}^{\infty} H^{s, \Psi}}\right),
\end{aligned}
$$

where in the last line we have used the embedding $H^{s, \Psi} \hookrightarrow H^{s}$. Combining this estimate with (4.10), Proposition 4.1-(a), and Proposition 4.6, we get

$$
\|\nabla v\|_{L^{\infty}} \leq \Phi_{4}(t)\left(1+t+\int_{0}^{t}\|\nabla v(\tau)\|_{L^{\infty}} d \tau\right) .
$$

By Gronwall's inequality this gives

$$
\|\nabla v(t)\|_{L^{\infty}} \leq \Phi_{5}(t) .
$$

Substituting this estimate into (4.10), we obtain finally

$$
f(t):=\|v\|_{\widetilde{L}_{t}^{\infty} H^{s, \Psi}}+\|\rho\|_{\widetilde{L}_{t}^{\infty} H^{s-2, \Psi}}+\|\rho\|_{\widetilde{L}_{t}^{1} H^{s, \Psi}} \leq \Phi_{6}(t) .
$$

This finishes the proof of the proposition.

\subsection{Inviscid limit}

We will prove that the family $\left(v_{\nu}, \rho_{\nu}\right)_{\nu>0}$ is converges strongly in $L_{T}^{\infty} H^{s} \times L_{T}^{\infty} H^{s-2}$ to the solution $(v, \rho)$ of the Euler-stratified system (1.6) as $\nu \rightarrow 0$. More precisely, we prove the following proposition.

Proposition 4.9. Let $s>5 / 2$, let $v^{0}$ be an axisymmetric divergence-free vector field such that $v^{0} \in H^{s}$, and let $\rho^{0} \in \chi_{m}^{s}$ with $6<m$. Then the solution $\left(v_{\nu}, \rho_{\nu}\right)$ to the system $(1.1)$ converges strongly in $L_{\mathrm{loc}}^{\infty}\left(\mathbb{R}_{+} ; H^{s}\right) \times L_{\mathrm{loc}}^{\infty}\left(\mathbb{R}_{+} ; H^{s-2}\right)$, as $\nu \rightarrow 0$, to the unique solution $(v, \rho)$ of the system (1.6).

More precisely, there exists $\Psi \in \mathcal{U}_{\infty}$ depending on the profile of the initial data and such that for every $T>0$

$$
\left\|v_{\nu}-v\right\|_{L_{T}^{\infty} H^{s}}+\left\|\rho_{\nu}-\rho\right\|_{L_{T}^{\infty} H^{s-2}} \leq\left(\sqrt{\nu}+\frac{1}{\Psi(\log (1 / \nu))}\right) \Phi_{7}(T) .
$$


Proof. We will proceed in two steps. In the first, we prove that for any fixed $T>0$, the family $\left(v_{\nu}, \rho_{\nu}\right)_{\nu}$ converges strongly in $L_{T}^{\infty} L^{2}$ when $\nu \rightarrow 0$, to the solution $(v, \rho)$ of the system (1.6) with initial data $\left(v^{0}, \rho^{0}\right)$. In the second step, we will show how to get strong convergence in the Sobolev spaces $L_{T}^{\infty} H^{s} \times L_{T}^{\infty} H^{s-2}$ with $s>5 / 2$. We set

$$
W_{\nu}:=v_{\nu}-v, \quad \Pi_{\nu}=p_{\nu}-p \text { and } \eta_{\nu}=\rho_{\nu}-\rho .
$$

Then we obtain the equations:

$$
\left\{\begin{aligned}
\partial_{t} W_{\nu}+v_{\nu} \cdot \nabla W_{\nu}+W_{\nu} \cdot \nabla v-\nu \Delta W_{\nu}+\nabla \Pi_{\nu} & =\nu \Delta v+\eta_{\nu} e_{z} \\
\partial_{t} \eta_{\nu}+v_{\nu} \cdot \nabla \eta_{\nu}-\Delta \eta_{\nu} & =-W_{\nu} \cdot \nabla \rho \\
\operatorname{div} W_{\nu} & =0 \\
\left(W_{\nu}, \eta_{\nu}\right)_{\mid t=0} & =0 .
\end{aligned}\right.
$$

First, we take the $L^{2}$ inner product of the first equation of (4.11) with $W_{\nu}$. Integrating by parts and using the Hölder inequality, we get

$$
\begin{aligned}
\frac{1}{2} \frac{d}{d t}\left\|W_{\nu}(t)\right\|_{L^{2}}^{2}+\nu\left\|\nabla W_{\nu}\right\|_{L^{2}}^{2} \leq & \nu\|\Delta v\|_{L^{2}}\left\|W_{\nu}\right\|_{L^{2}}+\|\nabla v\|_{L^{\infty}}\left\|W_{\nu}\right\|_{L^{2}}^{2} \\
& +\left\|\eta_{\nu}\right\|_{L^{2}}\left\|W_{\nu}\right\|_{L^{2}} .
\end{aligned}
$$

This gives

$$
\frac{d}{d t}\left\|W_{\nu}(t)\right\|_{L^{2}} \leq \nu\|\Delta v\|_{L^{2}}+\|\nabla v\|_{L^{\infty}}\left\|W_{\nu}\right\|_{L^{2}}+\left\|\eta_{\nu}\right\|_{L^{2}}
$$

Integrating in time this last inequality, we obtain

$$
\left\|W_{\nu}(t)\right\|_{L^{2}} \leq \nu\|\Delta v\|_{L_{t}^{1} L^{2}}+\left\|\eta_{\nu}\right\|_{L_{t}^{1} L^{2}}+\int_{0}^{t}\|\nabla v(\tau)\|_{L^{\infty}}\left\|W_{\nu}(\tau)\right\|_{L^{2}} d \tau
$$

From the inequality

$$
\|\Delta v\|_{L^{2}} \leq C\|v\|_{H^{s}}, \quad s \geq 2,
$$

and by using Gronwall's inequality and Proposition 4.7, we find

$$
\begin{aligned}
\left\|W_{\nu}\right\|_{L_{t}^{\infty} L^{2}} & \lesssim\left(\nu\|\Delta v\|_{L_{t}^{1} L^{2}}+\left\|\eta_{\nu}\right\|_{L_{t}^{1} L^{2}}\right) e^{\|\nabla v\|_{L_{t}^{1} L^{\infty}}} \\
& \leq\left(\nu t\|v\|_{\widetilde{L}_{t}^{\infty} H^{s}}+\left\|\eta_{\nu}\right\|_{L_{t}^{1} L^{2}}\right) \Phi_{6}(t) \leq\left(\nu+\left\|\eta_{\nu}\right\|_{L_{t}^{1} L^{2}}\right) \Phi_{6}(t) .
\end{aligned}
$$

There remains to estimate $\left\|\eta_{\nu}\right\|_{L_{t}^{1} L^{2}}$. To this end, we apply the maximum principle to the second equation of (4.11). We get

$$
\begin{aligned}
\left\|\eta_{\nu}(t)\right\|_{L^{2}} & \leq \int_{0}^{t}\left\|W_{\nu} \cdot \nabla \rho(\tau)\right\|_{L^{2}} d \tau \\
& \lesssim\left\|W_{\nu}\right\|_{L_{t}^{\infty} L^{2}}\|\nabla \rho\|_{L_{t}^{1} L^{\infty}} \leq \Phi_{4}(t)\left\|W_{\nu}\right\|_{L_{t}^{\infty} L^{2}},
\end{aligned}
$$

where we have used Proposition 4.6. Now, substituting (4.13) into (4.12) and using Gronwall's inequality we get, for all $t \in[0, T]$,

$$
\left\|W_{\nu}\right\|_{L_{t}^{\infty} L^{2}} \leq\left(\nu+\int_{0}^{t} \Phi_{4}(\tau)\left\|W_{\nu}\right\|_{L_{\tau}^{\infty} L^{2}} d \tau\right) \Phi_{6}(t) \leq \nu \Phi_{7}(t) .
$$


In view of (4.13), this gives that

$$
\left\|\eta_{\nu}\right\|_{L_{t}^{\infty} L^{2}} \leq \nu \Phi_{7}(t) \quad \forall t \in[0, T] .
$$

Therefore,

$$
\left\|W_{\nu}\right\|_{L_{t}^{\infty} L^{2}}+\left\|\eta_{\nu}\right\|_{L_{t}^{\infty} L^{2}} \leq \nu \Phi_{7}(t) \quad \forall t \in[0, T] .
$$

This proves the proof of the strong convergence in $L_{\mathrm{loc}}^{\infty}\left(\mathbb{R}_{+} ; L^{2}\right)$.

We now turn to the proof of the strong convergence in the Sobolev spaces. Let $M \in \mathbb{N}$, that will be chosen later. Then, by definition of the Sobolev space we have, for all $t \in \mathbb{R}_{+}$,

$$
\begin{aligned}
\left\|\left(v_{\nu}-v\right)(t)\right\|_{H^{s}}^{2}= & \sum_{q \leq M} 2^{2 q s}\left\|\Delta_{q}\left(v_{\nu}-v\right)(t)\right\|_{L^{2}}^{2}+\sum_{q>M} 2^{2 q s}\left\|\Delta_{q}\left(v_{\nu}-v\right)(t)\right\|_{L^{2}}^{2} \\
\lesssim & 2^{2 M s}\left\|\left(v_{\nu}-v\right)(t)\right\|_{L^{2}}^{2} \\
& +\frac{1}{\Psi^{2}(M)} \sum_{q>M} \Psi^{2}(q) 2^{2 q s}\left(\left\|\Delta_{q} v_{\nu}(t)\right\|_{L^{2}}+\left\|\Delta_{q} v(t)\right\|_{L^{2}}\right)^{2} \\
\lesssim & 2^{2 M s}\left\|W_{\nu}(t)\right\|_{L^{2}}^{2}+\frac{1}{\Psi^{2}(M)}\left(\left\|v_{\nu}(t)\right\|_{H^{s, \Psi}}^{2}+\|v(t)\|_{H^{s, \Psi}}^{2}\right) .
\end{aligned}
$$

We have used the fact that the profile $\Psi$ is nondecreasing. Now we use (4.14) and Proposition 4.7 to get

$$
\left\|\left(v_{\nu}-v\right)(t)\right\|_{H^{s}}^{2} \leq\left(2^{2 M s} \nu^{2}+\frac{1}{\Psi^{2}(M)}\right) \Phi_{7}(t) .
$$

It is enough to choose $M$ such that

$$
e^{2 M s} \approx \frac{1}{\nu}
$$

Therefore we obtain that

$$
\left\|\left(v_{\nu}-v\right)(t)\right\|_{H^{s}}^{2} \leq\left(\nu+\frac{1}{\Psi^{2}\left(\frac{1}{2 s} \log \left(\frac{1}{\nu}\right)\right)}\right) \Phi_{7}(t) .
$$

Similarly for $\left\|\left(\rho_{\nu}-\rho\right)(t)\right\|_{H^{s-2}}$, we obtain finally

$$
\left\|\left(\rho_{\nu}-\rho\right)(t)\right\|_{H^{s-2}}^{2} \leq\left(\nu+\frac{1}{\Psi^{2}\left(\frac{1}{2(s-2)} \log \left(\frac{1}{\nu}\right)\right)}\right) \Phi_{7}(t) \leq\left(\nu+\frac{1}{\Psi^{2}\left(\frac{1}{2 s} \log \left(\frac{1}{\nu}\right)\right)}\right) \Phi_{7}(t) .
$$

In the last identity we have used the fact that the profile $\Psi$ is nondecreasing. Now for any $\lambda>0$, the function defined by $\Psi_{\lambda}(x):=\Psi(\lambda x)$ belongs to the same class $\mathcal{U}_{\infty}$. Therefore, modifying $\Psi$ we get

$$
\left\|\left(v_{\nu}-v\right)(t)\right\|_{H^{s}}+\left\|\left(\rho_{\nu}-\rho\right)(t)\right\|_{H^{s-2}} \leq\left(\sqrt{\nu}+\frac{1}{\Psi\left(\log \left(\frac{1}{\nu}\right)\right)}\right) \Phi_{7}(t) .
$$

It follows that

$$
\left\|v_{\nu}-v\right\|_{L_{T}^{\infty} H^{s}}+\left\|\rho_{\nu}-\rho\right\|_{L_{T}^{\infty} H^{s-2}} \rightarrow 0 \quad \text { as } \nu \rightarrow 0 .
$$

This finishes the proof the proposition. 


\section{References}

[1] Abidi, H.: Résultats de régularité de solutions axisymétriques pour le système de Navier-Stokes. Bull. Sci. Math 132 (2008), no. 7, 592-624.

[2] Abidi, H., Hmidi, T. And Keranni, S.: On the global well-posedness for the axisymmetric Euler equations. Math. Ann. 347 (2010), no. 1, 15-41.

[3] Abidi, H., Hmidi, T. And KeraAni, S.: On the global reqularity of axisymmetric Navier-Stokes Boussinesq system. Discrete Contin. Dyn. Syst. 29 (2011), no. 3, 737-756.

[4] Bony, J. M.: Calcul symbolique et propagation des singularités pour les équations aux dérivées partielles non linéaires. Ann. Sci. Ecole Norm. Sup. (4) 14 (1981), no. 2, 209-246.

[5] Chemin, J. Y.: Perfect incompressible fluids. Oxford Lecture Series in Mathematics and its Applications 14, The Clarendon Press, Oxford University Press, New York, 1998.

[6] Danchin, R.: Axisymmetric incompressible flows with bounded vorticity. Russian. Math. Surveys 62 (2007), no. 3, 475-496.

[7] Fujita, F. and Kato, T.: On the nonstationary Navier-Stokes system. Rend. Sem. Mat. Univ. Padova 32 (1962), 243-260.

[8] Hmidi, T.: Régularité höldérienne des poches de tourbillon visqueuses. J. Math Pures Appl. (9) 84 (2005), no. 11, 1455-1495.

[9] Hmidi, T.: The low Mach number limit for the isentropic Euler system with axisymmetric initial data. J. Inst. Math. Jussieu 12 (2013), no. 2, 385-389.

[10] Hmidi, T. And Keranin, S.: On the global well-posedness of the Boussinesq system with zero viscosity. Indiana Univ. Math. J. 58 (2009), no. 4, 1591-1618.

[11] Hmidi, T., Keranin, S. And Rousset, F.: Global well-posedness for EulerBoussinesq system with critical dissipation. Comm. Partial Differential Equations 36 (2011), no. 3, 420-445.

[12] Hmidi, T. And Rousset, F.: Global well-posedness for the Euler-Boussinesq system with axisymmetric data. J. Funct. Anal. 260 (2011), no. 3, 745-796.

[13] Hmidi, T. And Sulaiman, S.: Incompressible limit for the 2D isentropic Euler system with critical initial data. To appear in Proc. Royal Soc. Edinburgh.

[14] Hmidi, T. And Zerguine, M.: Inviscid limit for axisymmetric Navier-Stokes system. Differential Integral Equations 22 (2009), no. 11-12, 1223-1246.

[15] Koch, H. and Tataru, D.: Well-posedness for the Navier-Stokes equations. Adv. Math. 157 (2001), no. 1, 22-35.

[16] Lemarié, P. G.: Recent developments in the Navier-Stokes problem. CRC Press, 2002.

[17] Leonardi, S., Málek, J., Nećas, J. And Pokorný, M.: On axially symmetric flows in $\mathbb{R}^{3}$. Z. Anal. Anwendungen 18 (1999), no. 3, 639-649.

[18] Leray, J.: Sur le mouvement d'un liquide visqueuse emplissant l'espace. Acta Math. 63 (1934), no. 1, 193-248.

[19] Majda, A.: Vorticity and the mathematical theory of an incompressible fluid flow. Comm. Pure Appl. Math. 39 (1986), no. S, suppl., S187-S220.

[20] Masmoudi, N.: Remarks about the inviscid limit of the Navier-Stokes system. Comm. Math. Phys. 270 (2007), no. 3, 777-788. 
[21] O'Neil, R.: Convolution operators and $L(p, q)$ spaces. Duke Math. J. 30 (1963), 129-142.

[22] Planchon, F.: Global stong solutions in Sobolev or Lebesgue spaces for the incompressible Navier-Stokes in $\mathbb{R}^{3}$. Ann. Inst. H. Poincaré Anal. Non linéaire 13 (1996), no. 3, 319-336.

[23] Shirota, T. and Yanagisawa, T.: Note on global existence for axially symmetric solutions of the Euler system. Proc. Japan Acad. Ser. A Math. Sci. 70 (1994), no. 10, 299-304.

[24] Sulaiman, S.: On the global existence for the axisymmetric Euler-Boussinesq system in critical Besov space. Asymptot. Anal. 77 (2012), no. 1-2, 89-121.

[25] Ukhovskit, M. R. And Iudovich, V. I.: Axially symmetric flows of ideal and viscous fluids filling the whole space. Prikl. Mat. Meh. 32 (1968), 59-69.

Received June 12, 2012.

Samira Lamin Sulaiman: IRMAR, Université de Rennes 1, Campus de Beaulieu, 35042 Rennes cedex, France.

E-mail: samira.sulaiman@voila.fr 